

\title{
Anabelian geometry and descent obstructions on moduli spaces
}

\author{
Stefan Patrikis, José Felipe Voloch and Yuri G. Zarhin
}

\begin{abstract}
We study the section conjecture of anabelian geometry and the sufficiency of the finite descent obstruction to the Hasse principle for the moduli spaces of principally polarized abelian varieties and of curves over number fields. For the former we show that the section conjecture fails and the finite descent obstruction holds for a general class of adelic points, assuming several well-known conjectures. This is done by relating the problem to a local-global principle for Galois representations. For the latter, we show how the sufficiency of the finite descent obstruction implies the same for all hyperbolic curves.
\end{abstract}

\section{Introduction}

Anabelian geometry is a program proposed by Grothendieck [1997a; 1997b] which suggests that for a certain class of varieties (called anabelian but, as yet, undefined) over a number field, one can recover the varieties from their étale fundamental group together with the Galois action of the absolute Galois group of the number field. Precise conjectures exist only for curves and some of them have been proved, notably by Mochizuki [1996]. Grothendieck suggested that moduli spaces of curves and abelian varieties (the latter perhaps less emphatically) should be anabelian. Already Ihara and Nakamura [1997] have shown that moduli spaces of abelian varieties should not be anabelian as one cannot recover their automorphism group from the fundamental group and we will further show that other anabelian properties fail in this case.

The finite descent obstruction is a construction that describes a subset of the adelic points of a variety over a number field containing the closure of the rational (or integral) points and is conjectured, for hyperbolic curves (Stoll [2007] in the projective case and Harari and Voloch [2010] in the affine case), to equal that closure. It's not unreasonable to conjecture the same for all anabelian varieties. The relationship between the finite descent obstruction and the section conjecture in anabelian geometry has been discussed by Harari and Stix [2012], Stix [2013,

MSC2010: primary 11G35; secondary 14G05, 14G35.

Keywords: Anabelian geometry, moduli spaces, abelian varieties, descent obstruction. 
Section 11], and others. We will review the relevant definitions below, although our point of view will be slightly different.

The purpose of this paper is to study the section conjecture of anabelian geometry and the finite descent obstruction for the moduli spaces of principally polarized abelian varieties and of curves over number fields. For the moduli of abelian varieties we show that the section conjecture fails in general and that both the section conjecture and finite descent obstruction hold for a general class of adelic points, assuming many established conjectures in arithmetic geometry (specifically, we assume the Hodge, Tate, Fontaine-Mazur and Grothendieck-Serre conjectures, in the precise forms stated in Section 3). This is done by converting the question into one about Galois representations.

The section conjecture predicts that sections of the fundamental exact sequence (Section 3, Equation (1)) of an anabelian variety over a number field correspond to rational points. In this paper, we look at the sections of the fundamental exact sequence of the moduli spaces of principally polarized abelian varieties that, locally at every place of the ground field, come from a point rational over the completion, which moreover is integral for all but finitely many places. This set is denoted $S_{0}\left(K, \mathcal{A}_{g}\right)$ and defined precisely at the end of Section 2. We explain, in Section 3, how sections of the fundamental exact sequence of the moduli spaces of principally polarized abelian varieties correspond to Galois representations and prove, Theorem 3.7, the following result.

Theorem 1.1. Assume the Hodge, Tate, Fontaine-Mazur, and Grothendieck-Serre conjectures. Let $K$ be a number field. Suppose $s \in S_{0}\left(K, \mathcal{A}_{g}\right)$ gives rise to a system of $\ell$-adic Galois representations one of which is absolutely irreducible. Then there exists, up to isomorphism, a unique principally polarized abelian variety which, viewed as point of $\mathcal{A}_{g}(K)$, induces (up to conjugation) the section $s$.

We also give examples (see Theorems 4.4 and 4.5) showing that weaker versions of the above result do not hold. Specifically, the local conditions cannot be weakened to hold almost everywhere, for instance.

For the moduli of curves, we show how combining some of our results and assuming sufficiency of finite descent obstruction for the moduli of curves, we deduce the sufficiency of finite descent obstruction for all hyperbolic curves.

In the next section we give more precise definitions of the objects we use and in the following two sections we give the applications mentioned above.

\section{Preliminaries}

Let $X / K$ be a smooth geometrically connected variety over a field $K$. Let $G_{K}$ be the absolute Galois group of $K$ and $\bar{X}$ the base-change of $X$ to an algebraic closure of $K$. We denote by $\pi_{1}(\cdot)$ the algebraic fundamental group functor on 
(geometrically pointed) schemes and we omit base-points from the notation. We have the fundamental exact sequence

$$
1 \rightarrow \pi_{1}(\bar{X}) \rightarrow \pi_{1}(X) \rightarrow G_{K} \rightarrow 1
$$

The map $p_{X}: \pi_{1}(X) \rightarrow G_{K}$ from the above sequence is obtained by functoriality from the structural morphism $X \rightarrow \operatorname{Spec} K$. Grothendieck's anabelian program is to specify a class of varieties, termed anabelian, for which the varieties and morphisms between them can be recovered from the corresponding fundamental groups together with the corresponding maps $p_{X}$ when the ground field is finitely generated over $\mathbb{Q}$. As this is very vague, we single out here two special cases with precise statements. The first is a (special case of a) theorem of Mochizuki [1996] which implies part of Grothendieck's conjectures for curves but also extends it by considering $p$-adic fields.

Theorem 2.1 [Mochizuki 1996]. Let X, Y be smooth projective curves of genus bigger than one over a field $K$ which is a subfield of a finitely generated extension of $\mathbb{Q}_{p}$. If there is an isomorphism from $\pi_{1}(X)$ to $\pi_{1}(Y)$ inducing the identity on $G_{K}$ via $p_{X}, p_{Y}$, then $X$ is isomorphic to $Y$.

A point $P \in X(K)$ gives, by functoriality, a section $G_{K} \rightarrow \pi_{1}(X)$ of the fundamental exact sequence (1) well-defined up to conjugation by an element of $\pi_{1}(\bar{X})$ (the indeterminacy is because of base points).

We denote by $H(K, X)$ the set of sections $G_{K} \rightarrow \pi_{1}(X)$ modulo conjugation by $\pi_{1}(\bar{X})$ and we denote by $\sigma_{X / K}: X(K) \rightarrow H(K, X)$ the map that associates to a point the class of its corresponding section, as above, and we call it the section map. As part of the anabelian program, it is expected that $\sigma_{X / K}$ is a bijection if $X$ is projective, anabelian and $K$ is finitely generated over its prime field. This is widely believed in the case of hyperbolic curves over number fields and is usually referred as the section conjecture. For a similar statement in the nonprojective case, one needs to consider the so-called cuspidal sections, see [Stix 2013, Section 18]. Although we will discuss nonprojective varieties in what follows, we will not need to specify the notion of cuspidal sections. The reason for this is that we will be considering sections that locally come from points (the Selmer set defined below) and these will not be cuspidal.

We remark that the choice of a particular section $s_{0}: G_{K} \rightarrow \pi_{1}(X)$ induces an action of $G_{K}$ on $\pi_{1}(\bar{X}), x \mapsto s_{0}(\gamma) x s_{0}(\gamma)^{-1}$. For an arbitrary section $s: G_{K} \rightarrow \pi_{1}(X)$ the map $\gamma \mapsto s(\gamma) s_{0}(\gamma)^{-1}$ is a 1-cocycle for the above action of $G_{K}$ on $\pi_{1}(\bar{X})$ and this induces a bijection $H^{1}\left(G_{K}, \pi_{1}(\bar{X})\right) \rightarrow H(K, X)$. We stress that this only holds when $H(K, X)$ is nonempty and a choice of $s_{0}$ can be made. It is possible for $H(K, X)$ to be empty, in which case there is no natural choice of action of $G_{K}$ 
on $\pi_{1}(\bar{X})$ by which to define $H^{1}\left(G_{K}, \pi_{1}(\bar{X})\right)$, which would be nonempty in any case, if defined.

Let $X / K$ be as above, where $K$ is now a number field. If $v$ is a place of $K$, we have the completion $K_{v}$ and a fixed inclusion $\bar{K} \subset \bar{K}_{v}$ induces a map $\alpha_{v}: G_{K_{v}} \rightarrow G_{K}$ and a map $\beta_{v}: \pi_{1}\left(X_{v}\right) \rightarrow \pi_{1}(X)$, where $X_{v}$ is the base-change of $X$ to $K_{v}$. We define the Selmer set of $X / K$ as the set $S(K, X) \subset H(K, X)$ consisting of the equivalence classes of sections $s$ such that for all places $v$ there exists $P_{v} \in X\left(K_{v}\right)$ with $s \circ \alpha_{v}=\beta_{v} \circ \sigma_{X_{v} / K_{v}}\left(P_{v}\right)$. Note that if $v$ is complex, then the condition at $v$ is vacuous and that if $v$ is real, $\sigma_{X_{v} / K_{v}}$ factors through $X\left(K_{v}\right)_{\bullet}$, the set of connected components of $X\left(K_{v}\right)$, equipped with the quotient topology (see [Mochizuki 2003; Pál 2011]). In the nonarchimedian case, $X\left(K_{v}\right)$ is totally disconnected so $X\left(K_{v}\right)=$ $X\left(K_{v}\right)_{\text {. }}$ and we have the following diagram:

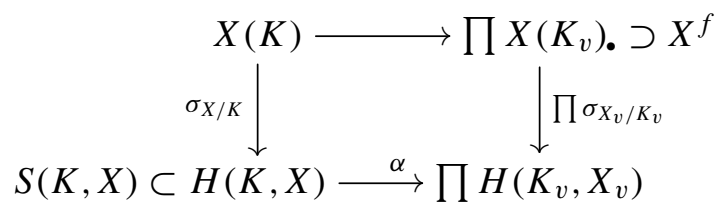

We define the set $X^{f}$ (the finite descent obstruction) as the set of points $\left(P_{v}\right)_{v} \in$ $\prod_{v} X\left(K_{v}\right)$. for which there exists $s \in H(K, X)$ (which is then necessarily an element of $S(K, X))$ satisfying $s \circ \alpha_{v}=\beta_{v} \circ \sigma_{X_{v} / K_{v}}\left(P_{v}\right)$ for all places $v$. Also, it is clear that the image of $X(K)$ is contained in $X^{f}$. At least when $X$ is proper, $X^{f}$ is closed (this follows from the compactness of $H(K, X)$ [Stix 2013, Corollary 45]). In that case, one may consider whether the closure of the image of $X(K)$ in $\prod X\left(K_{v}\right)$. equals $X^{f}$. A related statement is the equality $\sigma_{X / K}(X(K))=S(K, X)$, which is implied by the "section conjecture", i.e., the bijectivity of $\sigma_{X / K}: X(K) \rightarrow H(K, X)$. As a specific instance of this relation, we record the following easy fact.

Proposition 2.2. We have that $X^{f}=\varnothing$ if and only if $S(K, X)=\varnothing$.

Proof. If $X^{f} \neq \varnothing$ and $\left(P_{v}\right) \in X^{f}$, then there exists $s \in S(K, X)$ with $s \circ \alpha_{v}=$ $\beta_{v} \circ \sigma_{X_{v} / K_{v}}\left(P_{v}\right)$ for all places $v$, so $S(K, X) \neq \varnothing$.

If $s \in S(K, X)$, there exists $\left(P_{v}\right)$ with $s \circ \alpha_{v}=\beta_{v} \circ \sigma_{X_{v} / K_{v}}\left(P_{v}\right)$ for all places $v$. So $\left(P_{v}\right) \in X^{f}$.

If $X$ is not projective, then one has to take into account questions of integrality. We choose an integral model $\mathcal{X} / \mathcal{O}_{S, K}$, where $S$ is a finite set of places of $K$ and $\mathcal{O}_{S, K}$ is the ring of $S$-integers of $K$. The image of $X(K)$ in $X^{f}$ actually lands in the adelic points which are the points that satisfy $P_{v} \in \mathcal{X}\left(\mathcal{O}_{v}\right)$ for all but finitely many $v$, where $\mathcal{O}_{v}$ is the local ring at $v$. Similarly, the image of $\sigma_{X / K}$ belongs to the subset of $S(K, X)$ where the corresponding local points $P_{v}$ also belong to $\mathcal{X}\left(\mathcal{O}_{v}\right)$ for all but finitely many $v$. We denote this subset of $S(K, X)$ by $S_{0}(K, X)$ and call 
it the integral Selmer set. We note that $S_{0}(K, X)$ is independent of the choice of the model $\mathcal{X}$.

In order to set notation, we recall here some basic notions about the Tate module of abelian varieties which will be used in the next two sections. If $A$ is an abelian variety over the field $K$ then we write $\operatorname{End}(A)$ for its ring of all $K$-endomorphisms and $\operatorname{End}^{0}(A)$ for the corresponding (finite-dimensional semisimple) $\mathbb{Q}$-algebra $\operatorname{End}(A) \otimes \mathbb{Q}$. If $n \geq 3$ is an integer that is not divisible by $\operatorname{char}(K)$ and all points of order $n$ on $A$ are defined over $K$ then, by a theorem of Silverberg [1992], all $\bar{K}$-endomorphisms of $A$ are defined over $K$, i.e., lie in $\operatorname{End}(A)$.

If $\ell$ is a prime different from $\operatorname{char}(K)$ then we write $T_{\ell}(A)$ for the $\mathbb{Z}_{\ell}$-Tate module of $A$ which is a free $\mathbb{Z}_{\ell}$-module of $\operatorname{rank} 2 \operatorname{dim}(A)$ provided with the natural continuous homomorphism

$$
\rho_{\ell, A}: G_{K} \rightarrow \operatorname{Aut}_{\mathbb{Z}_{\ell}}\left(T_{\ell}(A)\right)
$$

and the $\mathbb{Z}_{\ell}$-ring embedding

$$
e_{l}: \operatorname{End}(A) \otimes \mathbb{Z}_{\ell} \hookrightarrow \operatorname{End}_{\mathbb{Z}_{\ell}}\left(T_{\ell}(A)\right) .
$$

The image of $\operatorname{End}(A) \otimes \mathbb{Z}_{\ell}$ commutes with $\rho_{\ell, A}\left(G_{K}\right)$. Tensoring by $\mathbb{Q}_{\ell}\left(\right.$ over $\left.\mathbb{Z}_{\ell}\right)$, we obtain the $\mathbb{Q}_{\ell}$-Tate module of $A$

$$
V_{\ell}(A)=T_{\ell}(A) \otimes_{\mathbb{Z}_{\ell}} \mathbb{Q}_{\ell},
$$

which is a $2 \operatorname{dim}(A)$-dimensional $\mathbb{Q}_{\ell}$-vector space containing

$$
T_{\ell}(A)=T_{\ell}(A) \otimes 1
$$

as a $\mathbb{Z}_{\ell}$-lattice. We may view $\rho_{\ell, A}$ as an $\ell$-adic representation

$$
\rho_{\ell, A}: G_{K} \rightarrow \operatorname{Aut}_{\mathbb{Z}_{\ell}}\left(T_{\ell}(A)\right) \subset \operatorname{Aut}_{\mathbb{Q}_{\ell}}\left(V_{\ell}(A)\right)
$$

and extend $e_{\ell}$ by $\mathbb{Q}_{\ell}$-linearity to the embedding of $\mathbb{Q}_{\ell}$-algebras

$$
\operatorname{End}^{0}(A) \otimes_{\mathbb{Q}} \mathbb{Q}_{\ell}=\operatorname{End}(A) \otimes \mathbb{Q}_{\ell} \hookrightarrow \operatorname{End}_{\mathbb{Q}_{\ell}}\left(V_{\ell}(A)\right),
$$

which we still denote by $e_{\ell}$. Further we will identify $\operatorname{End}^{0}(A) \otimes_{\mathbb{Q}} \mathbb{Q}_{\ell}$ with its image in $\operatorname{End}_{\mathbb{Q}_{\ell}}\left(V_{\ell}(A)\right)$.

This provides $V_{\ell}(A)$ with the natural structure of $G_{K}$-module; in addition, $\operatorname{End}^{0}(A) \otimes_{\mathbb{Q}} \mathbb{Q}_{\ell}$ is a $\mathbb{Q}_{\ell}$-(sub)algebra of endomorphisms of the Galois module $V_{\ell}(A)$. In other words,

$$
\operatorname{End}^{0}(A) \otimes_{\mathbb{Q}} \mathbb{Q}_{\ell} \subset \operatorname{End}_{G_{K}}\left(V_{\ell}(A)\right)
$$

Let $\chi_{\ell}$ be the cyclotomic character $\chi_{\ell}: G_{K} \rightarrow \mathbb{Z}_{\ell}^{*}$ that defines the Galois action on all $\ell$-power roots of unity, and $\mathbb{Z}_{\ell}(1)$ the $\ell$-adic Tate module of the multiplicative 
group $\mathbb{G}_{m}$. The group $\mathbb{Z}_{\ell}(1)$ is a free $\mathbb{Z}_{\ell}$-module of rank 1 provided with the Galois action that is defined by

$$
\chi_{\ell}: G_{K} \rightarrow \mathbb{Z}_{\ell}^{*}=\operatorname{Aut}_{\mathbb{Z}_{\ell}}\left(\mathbb{Z}_{\ell}(1)\right) .
$$

Let $\hat{A}$ be the dual (Picard) variety of $A$ [Lang 1959; Mumford 1970], which is an abelian variety over $K$ that is isogenous to $A$. There is the Weil pairing [Lang 1959, Chapter VII, Section 2]

$$
e_{\ell}: T_{\ell}(A) \times T_{\ell}(\hat{A}) \rightarrow \mathbb{Z}_{\ell}(1),
$$

which is a Galois-equivariant, $\mathbb{Z}_{\ell}$-bilinear perfect/unimodular pairing of free $\mathbb{Z}_{\ell^{-}}$ modules $T_{\ell}(A)$ and $T_{\ell}(\hat{A})$. This implies that the Galois modules $T_{\ell}(\hat{A})$ and $\operatorname{Hom}_{\mathbb{Z}_{\ell}}\left(T_{\ell}(A), \mathbb{Z}_{\ell}(1)\right)$ are isomorphic.

\section{Moduli of abelian varieties}

The moduli space of principally polarized abelian varieties of dimension $g$ is denoted by $\mathcal{A}_{g}$. It is actually a Deligne-Mumford stack or orbifold and we will consider its fundamental group as such. For a general definition of fundamental groups of stacks including a proof of the fundamental exact sequence in this generality, see [Zoonekynd 2001]. For a discussion of the case of $\mathcal{A}_{g}$, see [Hain 2011]. We can also get what we need from [Ihara and Nakamura 1997] (see below) or by working with a level structure which brings us back to the case of smooth varieties.

As $\mathcal{A}_{g}$ is defined over $\mathbb{Q}$, we can consider it over an arbitrary number field $K$. As per our earlier conventions, $\overline{\mathcal{A}}_{g}$ is the base change of $\mathcal{A}_{g}$ to an algebraic closure of $\mathbb{Q}$ and not a compactification. In fact, we will not consider a compactification at all here. The topological fundamental group of $\overline{\mathcal{A}}_{g}$ is the symplectic group $\operatorname{Sp}_{2 g}(\mathbb{Z})$ and the algebraic fundamental group is its profinite completion. When $g>1$ (which we henceforth assume) $\mathrm{Sp}_{2 g}(\mathbb{Z})$ has the congruence subgroup property [Bass et al. 1964; Mennicke 1965] and therefore its profinite completion is $\operatorname{Sp}_{2 g}(\hat{\mathbb{Z}})$.

The group $\pi_{1}\left(\mathcal{A}_{g}\right)$ is essentially described by the exact sequences (3.2) and (3.3) of [Ihara and Nakamura 1997] and it follows that the set $H\left(K, \mathcal{A}_{g}\right)$ consists of $\hat{\mathbb{Z}}$ representations of $G_{K}$ of rank $2 g$ preserving the symplectic form up to a multiplier given by the cyclotomic character. Indeed, it is clear that every section gives such a representation and the converse follows formally from the diagram below, which is a consequence of (3.2) and (3.3) of [Ihara and Nakamura 1997].

In the following we denote the cyclotomic character by $\chi: G_{K} \rightarrow \hat{\mathbb{Z}}^{*}$.

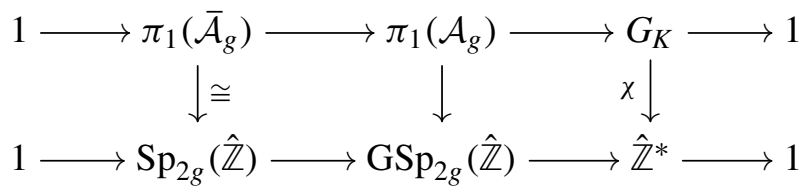


The coverings of $\overline{\mathcal{A}}_{g}$ corresponding to the congruence subgroups of $\mathrm{Sp}_{2 g}(\hat{\mathbb{Z}})$ are those obtained by adding level structures. In particular, for an abelian variety $A$, $\sigma_{\mathcal{A}_{g} / K}(A)=\prod T_{\ell}(A)$, the product of its Tate modules considered, as usual, as a $G_{K}$-module. If $K$ is a number field, whenever two abelian varieties are mapped to the same point by $\sigma_{\mathcal{A}_{g} / K}$, then they are isogenous, by [Faltings 1983]. The finiteness of isogeny classes of polarized abelian varieties over $K$ [Faltings 1983] (see also [Zarhin 1985]) implies that for any given $K$ and $g$ every fiber of $\sigma_{\mathcal{A}_{g} / K}$ is finite. On the other hand, $\sigma_{\mathcal{A}_{g} / K}$ is not necessarily injective to $S_{0}\left(K, \mathcal{A}_{g}\right)$. For example, for each $g$ there exists $K$ with noninjective $\sigma_{\mathcal{A}_{g} / K}$. Regarding surjectivity, we will prove that those elements of $S_{0}\left(K, \mathcal{A}_{g}\right)$ for which the corresponding Galois representation is absolutely irreducible (see below for the precise hypothesis and Theorem 3.7 for a precise statement) are in the image of $\sigma_{\mathcal{A}_{g} / K}$, assuming the Fontaine-Mazur conjecture, the Grothendieck-Serre conjecture on semisimplicity of $\ell$-adic cohomology of smooth projective varieties, and the Tate and Hodge conjectures. The integral Selmer set $S_{0}\left(K, \mathcal{A}_{g}\right)$, defined in the previous section, corresponds to the set of Galois representations that are almost everywhere unramified and, locally, come from abelian varieties (which thus are of good reduction for almost all places of $K$ ) and we will also consider a few variants of the question of surjectivity of $\sigma_{\mathcal{A}_{g} / K}$ to $S_{0}\left(K, \mathcal{A}_{g}\right)$ by different local hypotheses and discuss what we can and cannot prove. A version of this kind of question has also been considered by B. Mazur [1999].

Here is the setting. Let $K$ be a number field, with $G_{K}=\operatorname{Gal}(\bar{K} / K)$. Fix a finite set of rational primes $S$, and consider a collection of continuous $\ell$-adic representations

$$
\left\{\rho_{\ell}: G_{K} \rightarrow \mathrm{GL}_{N}\left(\mathbb{Q}_{\ell}\right)\right\}_{\ell \notin S} .
$$

We will say that the collection $\left\{\rho_{\ell}\right\}_{\ell \notin S}$ is weakly compatible if there exists a finite set of places $\Sigma$ of $K$ such that

(1) for all $\ell \notin S, \rho_{\ell}$ is unramified outside the union of $\Sigma$ and the places $\Sigma_{\ell}$ of $K$ dividing $\ell$; and

(2) for all $v \notin \Sigma \cup \Sigma_{\ell}$, denoting by $\mathrm{fr}_{v}$ a (geometric) frobenius element at $v$, the characteristic polynomial of $\rho_{\ell}\left(\mathrm{fr}_{v}\right)$ has rational coefficients and is independent of $\ell \notin S$. $^{1}$

Our aim is to prove the following:

Theorem 3.1. We will assume $\left\{\rho_{\ell}\right\}_{\ell \notin S}$ is weakly compatible and moreover satisfies the following three conditions:

(1) For some prime $\ell_{0} \notin S, \rho_{\ell_{0}}$ is de Rham at all places of $K$ above $\ell_{0}$.

(2) For some prime $\ell_{1} \notin S, \rho_{\ell_{1}}$ is absolutely irreducible.

${ }^{1}$ These systems were introduced by Serre [1989], who called them strictly compatible. 
(3) For some prime $\ell_{2} \notin S$, and at least one place $v \mid \ell_{2}$ of $K,\left.\rho_{\ell_{2}}\right|_{G_{K}}$ is de Rham with Hodge-Tate weights $-1,0$, each with multiplicity $N / 2$. (This condition holds if there exists an abelian variety $A_{v} / K_{v}$ such that $\left.\rho_{\ell_{2}}\right|_{G_{K_{v}}} \cong V_{\ell_{2}}\left(A_{v}\right)$.)

Assume the Hodge, Tate, Fontaine-Mazur, and Grothendieck-Serre conjectures, and suppose that the set $S$ is empty. Then there exists an abelian variety A over $K$ such that $\rho_{\ell} \cong V_{\ell}(A)$ for all $\ell$.

We note that the arguments allow $\ell_{0}=\ell_{2}$, and the reader may prefer to think of these together as a single condition; we have phrased it this way to have hypotheses that most clearly match the form of the argument.

We begin by making precise the combined implications of the GrothendieckSerre, Tate, and Fontaine-Mazur conjectures (the Hodge conjecture will only be used later, in the proof of Lemma 3.5). For any field $k$ and characteristic zero field $E$, let $\mathcal{M}_{k, E}$ denote the category of pure homological motives over $k$ with coefficients in $E$ (omitting $E$ from the notation will mean $E=\mathbb{Q}$ ).

Lemma 3.2. Assume the Tate conjecture for all finitely generated extensions $k$ of $\mathbb{Q}$. Then:

(1) The Lefschetz standard conjecture holds for all fields of characteristic zero.

(2) All of the standard conjectures (namely, the Künneth and Hodge standard conjectures, and the agreement of numerical and homological equivalence) hold for all fields of characteristic zero.

(3) For any field $k$ that can be embedded in $\mathbb{C}$, the category $\mathcal{M}_{k}$ is a semisimple neutral Tannakian category over $\mathbb{Q}$.

(4) For any finitely generated $k / \mathbb{Q}$, the étale $\ell$-adic realization functor

$$
\mathcal{M}_{k, \mathbb{Q}_{\ell}} \rightarrow \operatorname{Rep}_{\mathbb{Q}_{\ell}}\left(G_{k}\right),
$$

valued in the category of continuous $\ell$-adic representations of $G_{k}$, is fully faithful.

Proof. For the first assertion, see, e.g., [André 2004, 7.3.1.3]; for the second, see [André 2004, 5.4.2.2]. The third part is the basic motivating consequence of the standard conjectures (a fiber functor over $\mathbb{Q}$ is given by Betti cohomology, after fixing an embedding $k \hookrightarrow \mathbb{C}$ ): see [Jannsen 1992, Corollary 2], especially for the semisimplicity claim. Finally, for the last part, fullness is the Tate conjecture; and faithfulness follows from the agreement of numerical and homological equivalence and [Tate 1994, Lemma 2.5] (note that faithfulness on $\mathcal{M}_{k}$ is simply by definition of homological equivalence: it is only with $\mathbb{Q}_{\ell}$-coefficients that some argument is needed). 
For the rest of this section, we assume the Tate conjecture for all finitely generated $k$ of characteristic zero. Thus, we have a motivic Galois formalism: $\mathcal{M}_{k, E}$ is equivalent to $\operatorname{Rep}\left(\mathcal{G}_{k, E}\right)$ for some proreductive group $\mathcal{G}_{k, E}$ over $E$, the equivalence depending on the choice of an $E$-linear fiber functor. We will implicitly fix an embedding $k \hookrightarrow \mathbb{C}$ and use the associated Betti realization as our fiber functor. Before proceeding, we introduce two pieces of notation. For an extension of fields $k^{\prime} / k$, we denote the base-change of motives by

$$
\left.(\cdot)\right|_{k^{\prime}}: \mathcal{M}_{k, E} \rightarrow \mathcal{M}_{k^{\prime}, E} .
$$

This is not to be confused with the change of coefficients. Fix an embedding $\iota: \overline{\mathbb{Q}} \hookrightarrow \overline{\mathbb{Q}}_{\ell}$, so that when $E$ is a subfield of $\overline{\mathbb{Q}}$ we can speak of the $\ell$-adic realization

$$
H_{\iota}: \mathcal{M}_{k, E} \rightarrow \operatorname{Rep}_{\overline{\mathbb{Q}}_{\ell}}\left(G_{k}\right)
$$

associated to $\iota$.

Now we turn to the case of number fields, i.e., $k=K$. The Tate conjecture alone does not suffice to link Galois representations with motives: it yields full faithfulness of the $\ell$-adic realization (as in Lemma 3.2), but does not characterize the essential image. This is done via the combination of the Fontaine-Mazur and Grothendieck-Serre semisimplicity conjectures, which we now recall. A semisimple representation $r_{\ell}: G_{K} \rightarrow \mathrm{GL}_{N}\left(\mathbb{Q}_{\ell}\right)$ is said to be geometric (in the sense of Fontaine and Mazur [1995]) if it is unramified outside a finite set of places of $K$, and if for all $v \mid \ell$ of $K$, the restriction $\left.r_{\ell}\right|_{G_{K}}$ is de Rham (equivalently, potentially semistable, as in the original formulation). See [Fontaine and Ouyang 2007; Brinon and Conrad 2009] for the definition and basic properties of de Rham representations. Fontaine and Mazur have conjectured that any irreducible geometric $r_{\ell}$ is isomorphic to a subquotient of $H^{i}\left(X_{\bar{K}}, \mathbb{Q}_{\ell}\right)(j)$ for some smooth projective variety $X / K$ and some integers $i$ and $j$; that the converse assertion holds is a consequence of the base-change theorems of étale cohomology [SGA $4 \frac{1}{2}$ 1977] and the $p$-adic de Rham comparison isomorphism of Faltings [1989]. Grothendieck and Serre have moreover conjectured that for any smooth projective $X / K$, and any integer $i, H^{i}\left(X_{\bar{K}}, \mathbb{Q}_{\ell}\right)$ is a semisimple representation of $G_{K}$. Putting all of these conjectures together, we can characterize the essential image of $H_{l}$ :

Lemma 3.3. Assume the Tate, Fontaine-Mazur, and Grothendieck-Serre conjectures. Let $r_{\ell}: G_{K} \rightarrow \mathrm{GL}_{N}\left(\mathbb{Q}_{\ell}\right)$ be an irreducible geometric Galois representation. Then there exists an object $M$ of $\mathcal{M}_{K, \overline{\mathbb{Q}}}$ such that

$$
r_{\ell} \otimes_{\mathbb{Q}_{\ell}} \overline{\mathbb{Q}}_{\ell} \cong H_{l}(M)
$$

More generally, the essential image of $H_{\iota}$ consists of all semisimple geometric representations (with coefficients in $\overline{\mathbb{Q}}_{\ell}$ ) of $G_{K}$. 
Proof. The Fontaine-Mazur conjecture asserts that for some smooth projective variety $X / k, r_{\ell}$ is a subquotient of $H^{i}\left(X_{\bar{K}}, \mathbb{Q}_{\ell}\right)(j)$ for some integers $i$ and $j$, and the Grothendieck-Serre conjecture implies this subquotient is in fact a direct summand. Under the Künneth standard conjecture (a consequence of our hypotheses by Lemma 3.2), $\mathcal{M}_{K}$ has a canonical (weight) grading, and we denote by $H^{i}(X)$ the weight $i$ component of the motive of $X$. The Tate conjecture then implies (Lemma 3.2) that

$$
H_{l}: \operatorname{End}_{\mathcal{M}_{K}}\left(H^{i}(X)(j)\right) \otimes_{\mathbb{Q}} \overline{\mathbb{Q}}_{\ell} \stackrel{\sim}{\longrightarrow} \operatorname{End}_{\overline{\mathbb{Q}}_{\ell}\left[G_{K}\right]}\left(H^{i}\left(X_{\bar{K}}, \overline{\mathbb{Q}}_{\ell}\right)(j)\right)
$$

is an isomorphism.

Now, there is a projector (of $\overline{\mathbb{Q}}_{\ell}\left[G_{K}\right]$-modules) $H^{i}\left(X_{\bar{K}}, \overline{\mathbb{Q}}_{\ell}\right)(j) \rightarrow r_{\ell}$, which combined with Equation (2) yields a projector in $\operatorname{End}_{\mathcal{M}_{K}}\left(H^{i}(X)(j)\right) \otimes_{\mathbb{Q}} \overline{\mathbb{Q}}_{\ell}$ whose image has $\ell$-adic realization $r_{\ell}$. But $\operatorname{End}_{\mathcal{M}_{K}}\left(H^{i}(X)(j)\right)$ is a semisimple algebra over $\mathbb{Q}$ (Lemma 3.2), which certainly splits over $\overline{\mathbb{Q}}$, so the decomposition of $H^{i}(X)(j)$ into simple objects of $\mathcal{M}_{K, \overline{\mathbb{Q}}_{\ell}}$ is already realized in $\mathcal{M}_{K, \overline{\mathbb{Q}}}{ }^{2}$

For the final claim about the essential image (which we do not use in what follows), it suffices to show an irreducible $r_{\iota}: G_{K} \rightarrow \mathrm{GL}_{N}\left(\overline{\mathbb{Q}}_{\ell}\right)$ lies in the essential image. Such an $r_{l}$ is defined over a finite extension of $\mathbb{Q}_{\ell}$ and can thus be regarded as a higher-dimensional geometric representation $r_{\ell}$ with $\mathbb{Q}_{\ell}$-coefficients, necessarily semisimple. By the first part of the lemma, $r_{\ell} \otimes_{\mathbb{Q}_{\ell}} \overline{\mathbb{Q}}_{\ell}$ is isomorphic to $H_{l}(M)$ for some $M \in \mathcal{M}_{K, \overline{\mathbb{Q}}}$, and by the Tate conjecture there is a projector in $\operatorname{End}(M) \otimes_{\overline{\mathbb{Q}}} \overline{\mathbb{Q}}_{\ell}$ inducing the canonical (adjunction) projector $r_{\ell} \otimes_{\mathbb{Q}_{\ell}} \overline{\mathbb{Q}}_{\ell} \rightarrow r_{l}$. Arguing as before (a simple object of $\mathcal{M}_{K, \overline{\mathbb{Q}}_{\ell}}$ arises by scalar-extension from one of $\mathcal{M}_{K, \overline{\mathbb{Q}}}$ ), we see that $r_{\iota}$ is in the essential image of $H_{\iota}$.

Returning to our particular setting, fix any $\ell_{0} \notin S$ as in our first condition on the compatible system $\left\{\rho_{\ell}\right\}_{\ell \notin S}$, and also fix an embedding $\iota_{0}: \overline{\mathbb{Q}} \hookrightarrow \overline{\mathbb{Q}}_{\ell_{0}}$, so that Lemma 3.3 provides us with a number field (the linear combinations of correspondences needed to cut out a given object of $\mathcal{M}_{K, \overline{\mathbb{Q}}}$ have coefficients in a finite extension of $\mathbb{Q}$ ) $E \subset \overline{\mathbb{Q}}$ (which we may assume Galois over $\mathbb{Q}$ ) and a motivic Galois representation $\rho: \mathcal{G}_{K, E} \rightarrow \mathrm{GL}_{N, E}$ such that $H_{\iota_{0}}(\rho) \cong \rho_{\ell_{0}} \otimes \overline{\mathbb{Q}}_{\ell_{0}}$. Let us denote by $\lambda_{0}$ the place of $E$ induced by $E \subset \overline{\mathbb{Q}} \stackrel{\iota_{0}}{\longrightarrow} \overline{\mathbb{Q}}_{\ell}$. Then for all finite places $\lambda$ of $E$ (say $\lambda \mid \ell$ ), and for almost all places $v$ of $K$, compatibility gives us the following equality of rational numbers (note that $\rho_{\lambda}$ denotes the $\lambda$-adic realization of the motivic Galois representation $\rho$, while $\rho_{\ell}$ denotes the original $\ell$-adic representation in our compatible system):

$$
\operatorname{tr}\left(\rho_{\lambda}\left(\mathrm{fr}_{v}\right)\right)=\operatorname{tr}\left(\rho_{\lambda_{0}}\left(\mathrm{fr}_{v}\right)\right)=\operatorname{tr}\left(\rho_{\ell_{0}}\left(\mathrm{fr}_{v}\right)\right)=\operatorname{tr}\left(\rho_{\ell}\left(\mathrm{fr}_{v}\right) .\right.
$$

\footnotetext{
${ }^{2}$ In fact, it is realized over the maximal CM subfield of $\overline{\mathbb{Q}}$ : see, e.g., [Patrikis 2012, Lemma 4.1.22].
} 
Here we use the fact that the collection of $\ell$-adic realizations of a motive form a (weakly) compatible system; this follows from the Lefschetz trace formula, in its "formal" version for correspondences (see for instance [André 2004, 3.3.3, 7.1.4]). We deduce as usual (Brauer-Nesbitt and Chebotarev, see [Serre 1989, theorem on p. I-10; Ribet 1976, Theorem 1.3.1, p. 756]) that $\rho_{\ell}^{\text {ss }} \otimes_{\mathbb{Q}_{\ell}} E_{\lambda} \cong \rho_{\lambda}$; this holds for all $\lambda$ for which $\rho_{\ell}$ makes sense, i.e., for all $\lambda$ above $\ell \notin S$.

Recall that for some $\ell_{1} \notin S$, we have assumed $\rho_{\ell_{1}}$ is absolutely irreducible; hence for any place $\lambda_{1}$ of $E$ above $\ell_{1}$, the previous paragraph shows that $\rho_{\lambda_{1}} \cong \rho_{\ell_{1}} \otimes E_{\lambda_{1}}$ is absolutely irreducible. A fortiori, $\rho$ is absolutely irreducible, and then by the Tate conjecture all $\rho_{\lambda}$ are absolutely irreducible, so we can upgrade the conclusion of the previous paragraph to an isomorphism of absolutely irreducible representations $\rho_{\ell} \otimes_{\mathbb{Q}_{\ell}} E_{\lambda} \cong \rho_{\lambda}$, for all $\ell \notin S$.

The next question is whether having each (or almost all) $\rho_{\lambda}$ in fact definable over $\mathbb{Q}_{\ell}$ forces $\rho$ to be definable over $\mathbb{Q}$. Since the $\rho_{\lambda}$ descend to $\mathbb{Q}_{\ell}$, the Tate conjecture implies that for all $\sigma \in \operatorname{Gal}(E / \mathbb{Q}),{ }^{\sigma} \rho \cong \rho$; and since $\operatorname{End}(\rho)$ is $E$, the obstruction to descending $\rho$ to a $\mathbb{Q}$-rational representation of $\mathcal{G}_{K}$ is an element obs $\rho$ of $H^{1}\left(\mathrm{Gal}(E / \mathbb{Q}), \mathrm{PGL}_{N}(E)\right)$.

Lemma 3.4. With the notation above, $\mathrm{obs}_{\rho}$ in fact belongs to

$$
\operatorname{ker}\left(H^{1}\left(\operatorname{Gal}(E / \mathbb{Q}), \operatorname{PGL}_{N}(E)\right) \rightarrow \prod_{\ell \notin S} H^{1}\left(\operatorname{Gal}\left(E_{\lambda} / \mathbb{Q}_{\ell}\right), \operatorname{PGL}_{N}\left(E_{\lambda}\right)\right)\right)
$$

In particular, if $S$ is empty, then $\rho$ can be defined over $\mathbb{Q}$.

Proof. We know that each of the $\lambda$-adic realizations $\rho_{\lambda}$ (for $\lambda \mid \ell \notin S$ ) can be defined over $\mathbb{Q}_{\ell} ;$ to prove the lemma, we need to verify that the canonical localizations of $\operatorname{obs}_{\rho}$ (which arise by extending scalars on the motivic Galois representation) are in fact given by the corresponding obstruction classes for the $\lambda$-adic realizations. Thus, we have to recall how these realizations are constructed from $\rho$ itself. The surjection $\mathcal{G}_{K} \rightarrow G_{K}$ admits a continuous section on $\mathbb{Q}_{\ell}$-points, $s_{\ell}: G_{K} \rightarrow \mathcal{G}_{K}\left(\mathbb{Q}_{\ell}\right)$; composition with $\rho \otimes_{E} E_{\lambda}$ yields $\rho_{\lambda}$ :

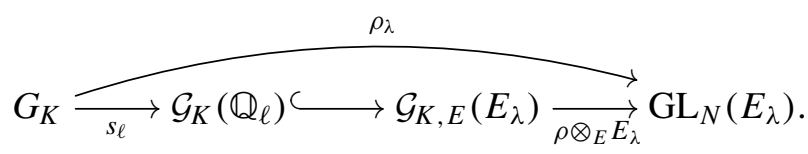

By construction of the respective obstruction classes, the canonical map from endomorphisms of $\rho \otimes_{E} E_{\lambda}$ to those of $\rho_{\lambda}$ realizes the obstruction class for $\rho_{\lambda}$ as the localization of $\operatorname{obs}_{\rho}$ at $\operatorname{Gal}\left(E_{\lambda} / \mathbb{Q}_{\ell}\right)$. But we have seen that $\rho_{\lambda}$ can be defined over $\mathbb{Q}_{\ell}$, so we conclude that obs $\rho$ has trivial restriction to each $\operatorname{Gal}\left(E_{\lambda} / \mathbb{Q}_{\ell}\right)$, as desired. 
For the final claim, note that by Hilbert 90 we can regard obs ${ }_{\rho}$ as an element of

$$
\operatorname{ker}\left(H^{2}\left(\operatorname{Gal}(E / \mathbb{Q}), E^{\times}\right) \rightarrow \prod_{\ell \notin S} H^{2}\left(\operatorname{Gal}\left(E_{\lambda} / \mathbb{Q}_{\ell}\right), E_{\lambda}^{\times}\right)\right) .
$$

If $S$ is empty, then the structure of the Brauer group of $\mathbb{Q}$ (which has only one infinite place!) then forces obs ${ }_{\rho}$ to be trivial.

Proof of Theorem 3.1. From now on we assume $S=\varnothing$, so that our compatible system $\left\{\rho_{\ell}\right\}_{\ell}$ arises from a rational representation

$$
\rho: \mathcal{G}_{K} \rightarrow \mathrm{GL}_{N, \mathbb{Q}} .
$$

Let $M$ be the rank $N$ object of $\mathcal{M}_{K}$ corresponding to $\rho$ via the Tannakian equivalence. Recall that we are given a prime $\ell_{2}$ and a place $v \mid \ell_{2}$ of $K$ for which we are given that $\left.\rho_{\ell_{2}}\right|_{G_{K}}$ is de Rham with Hodge numbers equal to those of an abelian variety of dimension $N / 2$. All objects of $\mathcal{M}_{K}$ enjoy the de Rham comparison theorem of " $\ell_{2}$-adic Hodge theory": denoting Fontaine's period ring over $K_{v}$ by $\mathrm{B}_{\mathrm{dR}, K_{v}}$, and the de Rham realization functor by $H_{\mathrm{dR}}: \mathcal{M}_{K} \rightarrow$ Fil $_{K}$ (the category of filtered $K$-vector spaces), we have the comparison (respecting filtration and $G_{K_{v}}$-action)

$$
H_{\mathrm{dR}}(M) \otimes_{K} \mathrm{~B}_{\mathrm{dR}, K_{v}} \stackrel{\sim}{\rightarrow} H_{\ell_{2}}(M) \otimes_{\mathbb{Q}_{2}} \mathrm{~B}_{\mathrm{dR}, K_{v}}
$$

hence

$$
H_{\mathrm{dR}}(M) \otimes_{K} K_{v} \cong \mathrm{D}_{\mathrm{dR}, K_{v}}\left(H_{\ell_{2}}(M)\right) .
$$

The Hodge filtration on $H_{\mathrm{dR}}(M)$ therefore satisfies

$$
\operatorname{dim}_{K} \operatorname{gr}^{0}\left(H_{\mathrm{dR}}(M)\right)=\operatorname{dim}_{K} \operatorname{gr}^{-1}\left(H_{\mathrm{dR}}(M)\right)=\frac{N}{2}
$$

and $\operatorname{gr}^{i}\left(H_{\mathrm{dR}}(M)\right)=0$ for $i \neq 0,-1$.

Now we turn to the Betti picture. Recall that to define the fiber functor on $\mathcal{M}_{K}$ we had to fix an embedding $K \hookrightarrow \mathbb{C}$; we regard $K$ as a subfield of $\mathbb{C}$ via this embedding. Then we also have the analytic Betti-de Rham comparison isomorphism

$$
H_{\mathrm{dR}}(M) \otimes_{K} \mathbb{C} \stackrel{\sim}{\longrightarrow} H_{\mathrm{B}}\left(\left.M\right|_{\mathbb{C}}\right) \otimes_{\mathbb{Q}} \mathbb{C} .
$$

We collect our findings in the following lemma, which relies on an application of the Hodge conjecture.

Lemma 3.5. There is an abelian variety $A$ over $K$, and an isomorphism of motives $H_{1}(A) \cong M$.

Proof. We see from Equations (3) and (4) that $H_{\mathrm{B}}\left(\left.M\right|_{\mathbb{C}}\right)$ is a polarizable rational Hodge structure of type $\{(0,-1),(-1,0)\}$. It follows from Riemann's theorem that there is an abelian variety $A / \mathbb{C}$ and an isomorphism of $\mathbb{Q}$-Hodge structures 
$H_{1}(A(\mathbb{C}), \mathbb{Q}) \cong H_{\mathrm{B}}\left(\left.M\right|_{\mathbb{C}}\right)$. The Hodge conjecture implies that this isomorphism comes from an isomorphism $\left.H_{1}(A) \stackrel{\sim}{\longrightarrow} M\right|_{\mathbb{C}}$ in $\mathcal{M}_{\mathbb{C}}$.

For any $\sigma \in \operatorname{Aut}(\mathbb{C} / \overline{\mathbb{Q}})$, we deduce an isomorphism

$$
\left.{ }^{\sigma} H_{1}(A) \stackrel{\sim}{\longrightarrow}{ }^{\sigma} M\right|_{\mathbb{C}}=\left.M\right|_{\mathbb{C}} \stackrel{\sim}{\longleftarrow} H_{1}(A),
$$

and again from Riemann's theorem we see that ${ }^{\sigma} A$ and $A$ are isogenous.

The following statement will be proven later in this paper.

Lemma 3.6. Let $\mathcal{K}$ be a countable subfield of the field $\mathbb{C}$ and $\overline{\mathcal{K}}$ the algebraic closure of $\mathcal{K}$ in $\mathbb{C}$. Let $\mathcal{Y}$ be a complex abelian variety of dimension $g$ such that for each field automorphism $\sigma \in \operatorname{Aut}(\mathbb{C} / \mathcal{K})$ the complex abelian variety $\mathcal{Y}$ and its "conjugate" $\sigma \mathcal{Y}=\mathcal{Y} \times \mathbb{C}, \sigma \mathbb{C}$ are isogenous. Then there exists an abelian variety $\mathcal{Y}_{0}$ over $\overline{\mathcal{K}}$ such that $\mathcal{Y}_{0} \times{ }_{\overline{\mathcal{K}}} \mathbb{C}$ is isomorphic to $\mathcal{Y}$.

It follows from Lemma 3.6 that $A$ has a model $A_{\overline{\mathbb{Q}}}$ over $\overline{\mathbb{Q}}$. The morphism

$$
\operatorname{Hom}_{\mathcal{M}_{\overline{\mathbb{Q}}}}\left(H_{1}\left(A_{\overline{\mathbb{Q}}}\right),\left.M\right|_{\overline{\mathbb{Q}}}\right) \rightarrow \operatorname{Hom}_{\mathcal{M}_{\mathbb{C}}}\left(H_{1}(A),\left.M\right|_{\mathbb{C}}\right)
$$

is an isomorphism, and then by general principles we deduce the existence of some finite extension $L / K$ inside $\overline{\mathbb{Q}}$ over which $A$ descends to an abelian variety $A_{L}$, and where we have an isomorphism $\left.H_{1}\left(A_{L}\right) \stackrel{\sim}{\rightarrow} M\right|_{L}$ in $\mathcal{M}_{L}$.

Finally, we treat the descent to $K$ itself. We form the restriction of scalars abelian variety $\operatorname{Res}_{L / K}\left(A_{L}\right)$; under the fully faithful embedding

$$
\mathrm{AV}_{K}^{0} \subset \mathcal{M}_{K}, \quad B \mapsto H_{1}(B),
$$

we can think of $H_{1}\left(\operatorname{Res}_{L / K}\left(A_{L}\right)\right)$ as $\operatorname{Ind}_{L}^{K}\left(H_{1}\left(A_{L}\right)\right)$, where the induction is taken in the sense of motivic Galois representations (note that the quotient $\mathcal{G}_{K} / \mathcal{G}_{L}$ is canonically $\operatorname{Gal}(L / K)$, so this is just the usual induction from a finite-index subgroup). Frobenius reciprocity then implies the existence of a nonzero map $M \rightarrow \operatorname{Ind}_{L}^{K}\left(H_{1}\left(A_{L}\right)\right)$ in $\mathcal{M}_{K}$. Since $M$ is a simple motive, this map realizes it as a direct summand in $\mathcal{M}_{K}$, and consequently (full-faithfulness) in $\mathrm{AV}_{K}^{0}$ as well. That is, there is an endomorphism of $\operatorname{Res}_{L / K}\left(A_{L}\right)$ whose image is an abelian variety $A$ over $K$ with $H_{1}(A) \cong M$.

Proof of Lemma 3.6. We may assume that $g \geq 1$. Since $\overline{\mathcal{K}}$ is also countable, we may replace $\mathcal{K}$ by $\overline{\mathcal{K}}$, i.e., assume that $\mathcal{K}$ is algebraically closed. Since the isogeny class of $\mathcal{Y}$ consists of a countable set of (complex) abelian varieties (up to an isomorphism), we conclude that the set $\operatorname{Aut}(\mathbb{C} / \mathcal{K})(\mathcal{Y})$ of isomorphism classes of complex abelian varieties of the form $\{\sigma \mathcal{Y} \mid \sigma \in \operatorname{Aut}(\mathbb{C} / \mathcal{K})\}$ is either finite or countable.

Our plan is as follows. Let us consider a fine moduli space $\mathcal{A}_{g}$ ? over $\overline{\mathbb{Q}}$ of $g$-dimensional abelian varieties (schemes) with certain additional structures (there should be only finitely many choices of these structures for any given abelian variety) such that it is a quasiprojective subvariety in some projective space $\mathbb{P}^{N}$. 
Choose these additional structures for $\mathcal{Y}$ (there should be only finitely many choices) and let $P \in \mathcal{A}_{g, ?}(\mathbb{C})$ be the corresponding point of our moduli space. We need to prove that

$$
P \in \mathcal{A}_{g, ?}(\mathcal{K}) .
$$

Suppose that it is not true. Then the orbit $\operatorname{Aut}(\mathbb{C} / \mathcal{K})(P)$ of $P$ is uncountable. Indeed, $P$ lies in one of the $(N+1)$ affine charts/spaces $\mathbb{A}^{N}$ that do cover $\mathbb{P}^{N}$. This implies that $P$ does not belong to $\mathbb{A}^{N}(\mathcal{K})$ and therefore (at least) one of its coordinates is transcendental over $\mathcal{K}$. But the $\operatorname{Aut}(\mathbb{C} / \mathcal{K})$-orbit of this coordinate coincides with uncountable $\mathbb{C} \backslash \mathcal{K}$ and therefore the $\operatorname{Aut}(\mathbb{C} / \mathcal{K})$-orbit $\operatorname{Aut}(\mathbb{C} / \mathcal{K})(P)$ of $P$ is uncountable in $\mathcal{A}_{g, ?}(\mathbb{C})$. However, for each $\sigma \in \operatorname{Aut}(\mathbb{C} / \mathcal{K})$ the point $\sigma(P)$ corresponds to $\sigma \mathcal{Y}$ with some additional structures and there are only finitely many choices for these structures. Since we know that the orbit $\operatorname{Aut}(\mathbb{C} / \mathcal{K})(\mathcal{Y})$ of $\mathcal{Y}$, is, at most, countable, we conclude that the orbit $\operatorname{Aut}(\mathbb{C} / \mathcal{K})(P)$ of $P$ is also, at most, countable, which is not the case. This gives us a desired contradiction.

We choose as $\mathcal{A}_{g}$ ? the moduli space of (polarized) abelian schemes of relative dimension $g$ with theta structures of type $\delta$ that was introduced and studied by D. Mumford [1966]. In order to choose (define) a suitable $\delta$, let us pick a totally symmetric ample invertible sheaf $\mathcal{L}_{0}$ on $\mathcal{Y}$ [Mumford 1966, Section 2] and consider its 8th power $\mathcal{L}:=\mathcal{L}_{0}^{8}$ in $\operatorname{Pic}(\mathcal{Y})$. Then $\mathcal{L}$ is a very ample invertible sheaf that defines a polarization $\Lambda(\mathcal{L})$ on $\mathcal{Y}$ [Mumford 1966, Part I, Section 1] that is an isogeny from $\mathcal{Y}$ to its dual; the kernel $H(\mathcal{L})$ of $\Lambda(\mathcal{L})$ is a finite commutative subgroup of $\mathcal{Y}(\mathbb{C})$ (that contains all points of order 8). The order of $H(\mathcal{L})$ is the degree of the polarization. The type $\delta$ is essentially the isomorphism class of the group $H(\mathcal{L})$ [Mumford 1966, Part I, Section 1, p. 294]. The resulting moduli space $\mathcal{A}_{g, ?}:=M_{\delta}$ [Mumford 1966, Part II, Section 6] enjoys all the properties that we used in the course of the proof.

Here is the anabelian application already mentioned in the introduction:

Theorem 3.7. Assume the Hodge, Tate, Fontaine-Mazur, and Grothendieck-Serre conjectures. Suppose $s \in S_{0}\left(K, \mathcal{A}_{g}\right)$ gives rise to a system of $\ell$-adic Galois representations one of which is absolutely irreducible. Then there exists up to isomorphism a unique principally polarized abelian variety $B / K$ with $\sigma_{\mathcal{A}_{g} / K}(B)=s$.

Proof. Let us write $s_{\ell}$ for the $\ell$-adic representation associated to $s$; thus $s_{\ell}$ is a representation of $G_{K}$ on a free $\mathbb{Z}_{\ell}$-module $\mathcal{T}_{\ell}$ of rank $2 g$, automatically satisfying Hypothesis 2 of Theorem 3.1 since $s$ belongs to $S_{0}\left(K, \mathcal{A}_{g}\right)$. Hypothesis 1 of Theorem 3.1 is satisfied by assumption, so we obtain an abelian variety $A / K$ (welldefined up to isogeny) whose rational Tate modules $V_{\ell}(A)$ are isomorphic (as $\ell$-adic representations) to the given $s_{\ell} \otimes_{\mathbb{Z}_{\ell}} \mathbb{Q}_{\ell}$ (for all $\ell$ ). Moreover Hypothesis 1 implies that the endomorphism ring of $A$ is $\mathbb{Z}$. It remains to see that within the isogeny class 
of $A$ there is a principally polarized abelian variety $B$ over $K$ whose integral Tate module $T_{\ell}(B)$ is isomorphic as a $\mathbb{Z}_{\ell}\left[G_{K}\right]$-module to $\mathcal{T}_{\ell}$ (for all $\ell$ ), i.e., such that $\sigma_{\mathcal{A}_{g} / K}(B)=s$. For this, we first observe that by [Deligne 1971, Proposition 3.3] (which readily generalizes to abelian varieties of any dimension), it suffices to show that for almost all $\ell$, there is an isomorphism $T_{\ell}(A) \cong \mathcal{T}_{\ell}$. Since $\operatorname{End}(A)=\mathbb{Z}$, [Zarhin 1985, Corollary 5.4.5] implies that $A[\ell]$ is an absolutely simple Galois module for almost all $\ell$, and hence that for almost all $\ell$, all Galois-stable lattices in $V_{\ell}(A)$ are of the form $\ell^{m} T_{\ell}(A)$ for some integer $m$; we conclude that $T_{\ell}(A)$ is isomorphic to $\mathcal{T}_{\ell}$ for almost all $\ell$. Thus there exists an abelian variety $B$ in the isogeny class of $A$ such that the $\mathbb{Z}_{\ell}\left[G_{K}\right]$-modules $T_{\ell}(B)$ and $\mathcal{T}_{\ell}$ are isomorphic for all $\ell$.

In order to prove the uniqueness of such a $B$ up to an isomorphism, first, notice that $\operatorname{End}(B)=\mathbb{Z}$. Second, let $C$ be an abelian variety over $K$ such that the $\mathbb{Z}_{\ell}\left[G_{K}\right]$ modules $T_{\ell}(B)$ and $T_{\ell}(C)$ are isomorphic for all primes $\ell$. This implies that the $\mathbb{Z}_{\ell}$-ranks of $T_{\ell}(B)$ and $T_{\ell}(C)$ coincide and therefore

$$
\operatorname{dim}(B)=\operatorname{dim}(C) .
$$

By a theorem of Faltings [1983],

$$
\operatorname{Hom}(B, C)=\operatorname{Hom}_{G_{K}}\left(T_{\ell}(B), T_{\ell}(C)\right) .
$$

Since $\operatorname{Hom}(B, C)$ is dense in $\operatorname{Hom}(B, C) \otimes \mathbb{Z}_{\ell}$ in the $\ell$-adic topology, and the set of isomorphisms $T_{\ell}(B) \cong T_{\ell}(C)$ is open in $\operatorname{Hom}(B, C) \otimes \mathbb{Z}_{\ell}$, there is a homomorphism $\phi_{\ell} \in \operatorname{Hom}(B, C)$ that induces an isomorphism of Tate modules $T_{\ell}(B) \cong T_{\ell}(C)$. Clearly, $\operatorname{ker}\left(\phi_{\ell}\right)$ does not contain points of order $\ell$ and therefore is finite. Since $\operatorname{dim}(B)=\operatorname{dim}(C)$, we obtain that $\phi_{\ell}$ is an isogeny, whose degree is prime to $\ell$. In particular, $B$ and $C$ are isogenous. On the other hand, since $\operatorname{End}(B)=\mathbb{Z}$, the group $\operatorname{Hom}(B, C)$ is a free $\mathbb{Z}$-module of rank 1 . Let us choose $\psi: B \rightarrow C$ that is a generator of $\operatorname{Hom}(B, C)$. Clearly, $\psi$ is an isogeny. Since for all primes $\ell$

$$
\phi_{\ell} \in \operatorname{Hom}(B, C)=\mathbb{Z} \cdot \psi,
$$

$\operatorname{deg}(\psi)$ is not divisible by $\ell$ and therefore $\operatorname{deg}(\psi)=1$, i.e., $\psi$ is an isomorphism of abelian varieties $B$ and $C$.

We still need to check that $B$ is principally polarized. Since $s_{\ell}$ comes from $s$, there is an alternating Galois-equivariant $\mathbb{Z}_{\ell}$-bilinear perfect/unimodular form

$$
\mathcal{T}_{\ell} \times \mathcal{T}_{\ell} \rightarrow \mathbb{Z}_{\ell}(1)
$$

Since $\mathcal{T}_{\ell}$ is isomorphic as a $\mathbb{Z}_{\ell}\left[G_{K}\right]$-module to $T_{\ell}(B)$, there is a Galois-equivariant, $\mathbb{Z}_{\ell}$-bilinear perfect/unimodular form

$$
T_{\ell}(B) \times T_{\ell}(B) \rightarrow \mathbb{Z}_{\ell}(1) .
$$


This implies that the Galois modules $T_{\ell}(B)$ and $\operatorname{Hom}_{\mathbb{Z}_{\ell}}\left(T_{\ell}(B), \mathbb{Z}_{\ell}(1)\right)$ are isomorphic. It follows from the last sentence of Section 2 that the Galois modules $T_{\ell}(B)$ and $T_{\ell}(\hat{B})$ are isomorphic for all primes $\ell$. This implies that the abelian varieties $\hat{B}$ and $B$ are isomorphic. Since $\operatorname{End}(B)=\mathbb{Z}$, there is an isomorphism $\mu: B \rightarrow \hat{B}$ such that $\operatorname{Hom}(B, \hat{B})=\mathbb{Z} \cdot \mu$. Let $\lambda: B \rightarrow \hat{B}$ be a polarization on $B$. Then there is a nonzero integer $n$ such that $\lambda=n \cdot \mu$. Replacing if necessary $\mu$ by $-\mu$, we may and will assume that $n$ is a positive integer. It follows from [Mumford 1970, Section 23, Theorem 3] that $\mu$ is a polarization, which is obviously principal. (Clearly, there is exactly one principal polarization on $B$, namely $\mu$.) So, $\sigma_{\mathcal{A}_{g} / K}(B)$ is defined and obviously coincides with $s$.

Remark 3.8. Note that for each prime $\ell$ we get the Riemann form [Lang 1959, Chapter VII, Section 2; Mumford 1970, Section 20]

$$
E_{\ell, \mu}: T_{\ell}(B) \times T_{\ell}(B) \rightarrow \mathbb{Z}_{\ell}(1), \quad x, y \mapsto e_{\ell}(x, \mu y) \quad \text { for all } x, y \in T_{\ell}(B),
$$

which is an alternating Galois-equivariant $\mathbb{Z}_{\ell}$-bilinear perfect/unimodular form on the free $\mathbb{Z}_{\ell}$-module $T_{\ell}(B)$. Since $\operatorname{End}(B)=\mathbb{Z}$, the already cited result of Faltings implies that $\operatorname{End}_{G_{K}}\left(T_{\ell}(A)\right)=\mathbb{Z}_{\ell}$. It follows that any alternating Galois-equivariant $\mathbb{Z}_{\ell}$-bilinear perfect/unimodular form

$$
T_{\ell}(B) \times T_{\ell}(B) \rightarrow \mathbb{Z}_{\ell}(1)
$$

coincides with $c_{\ell} \cdot E_{\ell, \mu}$ for some $c_{\ell} \in \mathbb{Z}_{\ell}^{*}$. This implies that any isomorphism between the $\mathbb{Z}_{\ell}\left[G_{K}\right]$-modules $\mathcal{T}_{\ell}$ and $T_{\ell}(B)$ induces isomorphisms between the corresponding symplectic groups and between the corresponding groups of symplectic similitudes.

Results in the same vein as this corollary have been obtained for elliptic curves over $\mathbb{Q}$ in [Helm and Voloch 2011] and for elliptic curves over function fields in [Voloch 2012].

\section{Counterexamples}

Now we will construct an example of Galois representation that will provide us with examples that show that some of the hypotheses of the above results are indispensable.

Let $k$ be a real quadratic field. Let us choose a prime $p$ that splits in $k$. Now let $D$ be the indefinite quaternion $k$-algebra that splits everywhere outside (two) prime divisors of $p$ and is ramified at these divisors. If $\ell$ is a prime then we have

$$
D \otimes_{\mathbb{Q}} \mathbb{Q}_{\ell}=\left[D \otimes_{k} k\right] \otimes_{\mathbb{Q}} \mathbb{Q}_{\ell}=D \otimes_{k}\left[k \otimes_{\mathbb{Q}} \mathbb{Q}_{\ell}\right] .
$$

This implies that if $\ell \neq p$ then $D \otimes_{\mathbb{Q}} \mathbb{Q}_{\ell}$ is either (isomorphic to) the simple matrix algebra (of size 2 ) over a quadratic extension of $\mathbb{Q}_{\ell}$ or a direct sum of two copies of 
the simple matrix algebra (of size 2 ) over $\mathbb{Q}_{\ell}$. (In both cases, $D \otimes_{\mathbb{Q}} \mathbb{Q}_{\ell}$ is isomorphic to the matrix algebra $\mathbb{M}_{2}\left(k \otimes_{\mathbb{Q}} \mathbb{Q}_{\ell}\right)$ of size 2 over $k \otimes_{\mathbb{Q}} \mathbb{Q}_{\ell}$.)

In particular, the image of $D \otimes_{\mathbb{Q}} \mathbb{Q}_{\ell}$ under each nonzero $\mathbb{Q}_{\ell}$-algebra homomorphism contains zero divisors.

Let $Y$ be an abelian variety over a field $L$. Suppose that all $\bar{L}$-endomorphisms of $Y$ are defined over $L$ and there is a $\mathbb{Q}$-algebra embedding

$$
D \hookrightarrow \operatorname{End}^{0}(Y)
$$

that sends 1 to 1 . This gives us the embedding

$$
D \otimes_{\mathbb{Q}} \mathbb{Q}_{\ell} \subset \operatorname{End}^{0}(Y) \otimes_{\mathbb{Q}} \mathbb{Q}_{\ell} \subset \operatorname{End}_{G_{L}}\left(V_{\ell}(Y)\right) .
$$

Recall that if $\ell \neq p$ then $D \otimes_{\mathbb{Q}} \mathbb{Q}_{\ell}$ is isomorphic to the matrix algebra of size 2 over $k \otimes_{\mathbb{Q}} \mathbb{Q}_{\ell}$. This implies that there are two isomorphic $\mathbb{Q}_{\ell}\left[G_{L}\right]$-submodules $W_{1, \ell}(Y)$ and $W_{2, \ell}(Y)$ in $V_{\ell}(Y)$ such that

$$
V_{\ell}(Y)=W_{1, \ell}(Y) \oplus W_{2, \ell}(Y) \cong W_{1, \ell}(Y) \oplus W_{1, \ell}(Y) \cong W_{2, \ell}(Y) \oplus W_{2, \ell}(Y) .
$$

If we denote by $W_{\ell}(Y)$ the $\mathbb{Q}_{\ell}\left[G_{L}\right]$-module $W_{1, \ell}$ then we get an isomorphism of $\mathbb{Q}_{\ell}\left[G_{L}\right]$-modules

$$
V_{\ell}(Y) \cong W_{\ell}(Y) \oplus W_{\ell}(Y) .
$$

This implies that the centralizer $\operatorname{End}_{G_{L}}\left(V_{\ell}(Y)\right)$ coincides with the matrix algebra $\mathbb{M}_{2}\left(\operatorname{End}_{G_{L}}\left(W_{\ell}(Y)\right)\right)$ of size 2 over the centralizer $\operatorname{End}_{G_{L}}\left(W_{\ell}(Y)\right)$.

If $\ell=p$ then $k \otimes_{\mathbb{Q}} \mathbb{Q}_{p}=\mathbb{Q}_{p} \oplus \mathbb{Q}_{p}$ and $D \otimes_{\mathbb{Q}} \mathbb{Q}_{p}$ splits into a direct sum of two (mutually isomorphic) quaternion algebras over $\mathbb{Q}_{p}$. This also gives us a splitting of the Galois module $V_{p}(Y)$ into a direct sum

$$
V_{p}(Y)=W_{1, p}(Y) \oplus W_{2, p}(Y) .
$$

of its certain nonzero $\mathbb{Q}_{p}\left[G_{L}\right]$-submodules $W_{1, p}(Y)$ and $W_{2, p}(Y)$. (Actually,

$$
\operatorname{dim}_{\mathbb{Q}_{p}} W_{1, p}=\operatorname{dim}_{\mathbb{Q}_{p}} W_{2, p}=\operatorname{dim}(Y),
$$

because $V_{p}(Y)$ is a free $k \otimes_{\mathbb{Q}} \mathbb{Q}_{p}$-module of $\operatorname{rank} 2 \operatorname{dim}(Y) /[k: \mathbb{Q}]=\operatorname{dim}(Y)$ [Ribet 1976, Theorem 2.1.1 on p. 768].)

Remark. Let $L$ be a finitely generated field of characteristic 0. Suppose that $D=\operatorname{End}^{0}(Y)$. By Faltings' results $[1983 ; 1984]$ about the Galois action on Tate modules of abelian varieties, the $G_{L}$-module $V_{\ell}(Y)$ is semisimple and

$$
\operatorname{End}_{G_{L}}\left(V_{\ell}(Y)\right)=D \otimes_{\mathbb{Q}} \mathbb{Q}_{\ell} .
$$

This implies that if $\ell \neq p$ then (the submodule) $W_{\ell}(Y)$ is also semisimple and

$$
\mathbb{M}_{2}\left(\operatorname{End}_{G_{L}}\left(W_{\ell}(Y)\right)\right) \cong \mathbb{M}_{2}\left(k \otimes_{\mathbb{Q}} \mathbb{Q}_{\ell}\right) \text {. }
$$


It follows that

$$
\operatorname{End}_{G_{L}}\left(W_{\ell}(Y)\right) \cong k \otimes_{\mathbb{Q}} \mathbb{Q}_{\ell} .
$$

On the other hand, the $G_{L}$-modules $W_{1, p}(Y)$ and $W_{2, p}(Y)$ are nonisomorphic.

According to Shimura [1963] (see also the case of Type II $\left(e_{0}=2\right)$ with $m=1$ in [Oort 1988, Table 8.1 on p. 498] and [Oort and Zarhin 1995, table on p. 23]), there exists a complex abelian fourfold $X$, whose endomorphism algebra $\operatorname{End}^{0}(X)$ is isomorphic to $D$. Clearly, $X$ is defined over a finitely generated field of characteristic zero. It follows from Serre's variant of Hilbert's irreducibility theorem for infinite Galois extensions combined with results of Faltings that there exists a number field $K$ and an abelian fourfold $A$ over $K$ such that the endomorphism algebra of all $\bar{K}$-endomorphisms of $A$ is also isomorphic to $D$ (see [Noot 1995, Corollary 1.5 on p. 165]). Enlarging $K$, we may assume that all points of order 12 on $A$ are defined over $K$ and therefore, by the theorem of Silverberg, all $\bar{K}$-endomorphisms of $A$ are defined over $K$. Now Raynaud's criterion [SGA 7 1 1972] (see also [Silverberg and Zarhin 1995]), implies that $A$ has everywhere semistable reduction. On the other hand,

$$
\operatorname{dim}_{\mathbb{Q}} \operatorname{End}^{0}(A)=\operatorname{dim}_{\mathbb{Q}} D=8>4=\operatorname{dim}(A) .
$$

By [Oort 1988, Lemma 3.9 on p. 484], $A$ has everywhere potential good reduction. This implies that $A$ has good reduction everywhere. If $v$ is a nonarchimedean place of $K$ with finite residue field $\kappa(v)$ then we write $A(v)$ for the reduction of $A$ at $v$; clearly, $A(v)$ is an abelian fourfold over $\kappa(v)$. If $\operatorname{char}(\kappa(v)) \neq 2$ then all points of order 4 on $A(v)$ are defined over $\kappa(v)$; if $\operatorname{char}(\kappa(v)) \neq 3$ then all points of order 3 on $A(v)$ are defined over $\kappa(v)$. It follows from the theorem of Silverberg that all $\overline{\kappa(v)}$-endomorphisms of $A(v)$ are defined over $\kappa(v)$. For each $v$ we get an embedding of $\mathbb{Q}$-algebras

$$
D \cong \operatorname{End}^{0}(A) \hookrightarrow \operatorname{End}^{0}(A(v)) .
$$

In particular, $\operatorname{End}^{0}(A(v))$ is a noncommutative $\mathbb{Q}$-algebra, whose $\mathbb{Q}$-dimension is divisible by 8 .

Theorem 4.1. If $\ell:=\operatorname{char}(\kappa(v)) \neq p$ then $A(v)$ is not simple over $\kappa(v)$.

Proof. We write $q_{v}$ for the cardinality of $\kappa(v)$. Clearly, $q_{v}$ is a power of $\ell$.

Suppose that $A(v)$ is simple over $\kappa(v)$. Since all endomorphisms of $A(v)$ are defined over $\kappa(v)$, the abelian variety $A(v)$ is absolutely simple.

Let $\pi$ be a Weil $q_{v}$-number that corresponds to the $\kappa(v)$-isogeny class of $A(v)$ [Tate 1966; 1971]. In particular, $\pi$ is an algebraic integer (complex number), all whose Galois conjugates have (complex) absolute value $\sqrt{q_{v}}$. In particular, the product

$$
\pi \bar{\pi}=q_{v},
$$


where $\bar{\pi}$ is the complex conjugate of $\pi$.

Let $E=\mathbb{Q}(\pi)$ be the number field generated by $\pi$ and let $\mathcal{O}_{E}$ be the ring of integers in $E$. Then $E$ contains $\bar{\pi}$ and is isomorphic to the center of $\operatorname{End}^{0}(A(v))$ [Tate 1966; 1971]; one may view $\operatorname{End}^{0}(A(v))$ as a central division algebra over $E$. It is known that $E$ is either $\mathbb{Q}, \mathbb{Q}(\sqrt{\ell})$ or a (purely imaginary) CM field [Tate 1971, p. 97]. It is known [ibid] that in the first two (totally real) cases simple $A(v)$ has dimension 1 or 2, which is not the case. So, $E$ is a CM field; $\operatorname{Since} \operatorname{dim}(A(v))=4$ and $[E: \mathbb{Q}]$ divides $2 \operatorname{dim}(A(v))$, we have $[E: \mathbb{Q}]=2,4$ or 8 . By [Tate 1971, p. 96 , Theorem 1(ii), formula (2) $]^{3}$,

$$
8=2 \cdot 4=2 \operatorname{dim}(A(v)))=\sqrt{\operatorname{dim}_{E}\left(\operatorname{End}^{0}(A(v))\right.} \cdot[E: \mathbb{Q}] .
$$

Since $\operatorname{End}^{0}(A(v))$ is noncommutative, it follows that $E$ is either an imaginary quadratic field and $\operatorname{End}^{0}(A(v))$ is a 16-dimensional division algebra over $E$ or $E$ is a $\mathrm{CM}$ field of degree 4 and $\operatorname{End}^{0}(A(v))$ is a 4-dimensional (i.e., quaternion) division algebra over $E$. In both cases $\operatorname{End}^{0}(A(v))$ is unramified at all places of $E$ except some places of residual characteristic $\ell$ [Tate 1971, p. 96, Theorem 1(ii)]. It follows from the Hasse-Brauer-Noether theorem that $\operatorname{End}^{0}(A(v))$ is ramified at, at least, two places of $E$ with residual characteristic $\ell$. This implies that $\mathcal{O}_{E}$ contains, at least, two maximal ideals that lie above $\ell$.

Clearly,

$$
\pi, \bar{\pi} \in \mathcal{O}_{E} .
$$

Recall that $\pi \bar{\pi}=q_{v}$ is a power of $\ell$. This implies that for every prime $r \neq \ell$ both $\pi$ and $\bar{\pi}$ are $r$-adic units in $E$.

First assume that $E$ has degree 4 and $\operatorname{End}^{0}(A(v))$ is a quaternion algebra. Then (thanks to the theorem of Hasse-Brauer-Noether) there exists a place $w$ of $E$ with residual characteristic $\ell$ and such that the localization $\operatorname{End}^{0}(A(v)) \otimes_{E} E_{w}$ is a quaternion division algebra over the $w$-adic field $E_{w}$. On the other hand, there is a nonzero (because it sends 1 to 1$) \mathbb{Q}_{\ell}$-algebra homomorphism

$$
D \otimes_{\mathbb{Q}} \mathbb{Q}_{\ell} \rightarrow \operatorname{End}^{0}(A(v)) \otimes_{\mathbb{Q}} \mathbb{Q}_{\ell} \rightarrow \operatorname{End}^{0}(A(v)) \otimes_{E} E_{w} .
$$

This implies that $\operatorname{End}^{0}(A(v)) \otimes_{E} E_{w}$ contains zero divisors, which is not the case and we get a contradiction.

So, now we assume that $E$ is an imaginary quadratic field and

$$
\operatorname{dim}_{E}\left(\operatorname{End}^{0}(A(v))\right)=16=4^{2} .
$$

In particular, the order of the class of $\operatorname{End}^{0}(A(v))$ in the Brauer group of $E$ divides 4 and therefore is either 2 or 4 .

\footnotetext{
${ }^{3}$ In [Tate 1971] our $E$ is denoted by $F$ while our $\operatorname{End}^{0}(A(v))$ is denoted by $E$.
} 
We have already seen that there exist, at least, two maximal ideals in $\mathcal{O}_{E}$ that lie above $\ell$. Since $E$ is an imaginary quadratic field, the ideal $\ell \mathcal{O}_{L}$ of $\mathcal{O}_{L}$ splits into a product of two distinct complex-conjugate maximal ideals $w_{1}$ and $w_{2}$ and therefore

$$
E_{w_{1}}=\mathbb{Q}_{\ell}, \quad E_{w_{2}}=\mathbb{Q}_{\ell} ; \quad\left[E_{w_{1}}: \mathbb{Q}_{\ell}\right]=\left[E_{w_{2}}: \mathbb{Q}_{\ell}\right]=1 .
$$

Let

$$
\operatorname{ord}_{w_{i}}: E^{*} \rightarrow \mathbb{Z}
$$

be the discrete valuation map that corresponds to $w_{i}$. Recall that $q_{v}$ is a power of $\ell$, i.e., $q_{v}=\ell^{N}$ for a certain positive integer $N$. Clearly

$$
\operatorname{ord}_{w_{i}}(\ell)=1, \quad \operatorname{ord}_{w_{i}}(\pi)+\operatorname{ord}_{w_{i}}(\bar{\pi})=\operatorname{ord}_{w_{i}}\left(q_{v}\right)=N .
$$

By [Tate 1971, p. 96, Theorem 1(ii), formula (1)], the local invariant of $\operatorname{End}^{0}(A(v))$ at $w_{i}$ is

$$
\frac{\operatorname{ord}_{w_{i}}(\pi)}{\operatorname{ord}_{w_{i}}\left(q_{v}\right)} \cdot\left[E_{w_{i}}: \mathbb{Q}_{\ell}\right](\bmod 1)=\frac{\operatorname{ord}_{w_{i}}(\pi)}{N}(\bmod 1) .
$$

In addition, the sum in $\mathbb{Q} / \mathbb{Z}$ of local invariants of $\operatorname{End}^{0}(A(v))$ at $w_{1}$ and $w_{2}$ is zero [Tate 1971, Section 1, Theorem 1 and Example b)]; we have already seen that its local invariants at all other places of $E$ do vanish. Using the Hasse-BrauerNoether theorem and taking into account that the order of the class of $\operatorname{End}^{0}(A(v))$ in the Brauer group of $E$ is either 2 or 4 , we conclude that the local invariants of $\operatorname{End}^{0}(A(v))$ at $\left\{w_{1}, w_{2}\right\}$ are either $\left\{\frac{1}{4} \bmod 1, \frac{3}{4} \bmod 1\right\}$ or $\left\{\frac{3}{4} \bmod 1, \frac{1}{4} \bmod 1\right\}$ (and in both cases the order of $\operatorname{End}^{0}(A(v))$ in the Brauer group of $E$ is 4) or $\left\{\frac{1}{2} \bmod 1, \frac{1}{2} \bmod 1\right\}$. In the latter case it follows from the formula for the $w_{i}$-adic invariant of $\operatorname{End}^{0}(A(v))$ that

$$
\operatorname{ord}_{w_{i}}(\pi)=\frac{N}{2}=\operatorname{ord}_{w_{i}}(\bar{\pi})
$$

and therefore $\bar{\pi} / \pi$ is a $w_{i}$-adic unit for both $w_{1}$ and $w_{2}$. Therefore $\bar{\pi} / \pi$ is an $\ell$-adic unit. This implies that $\bar{\pi} / \pi$ is a unit in imaginary quadratic $E$ and therefore is a root of unity. It follows that

$$
\frac{\pi^{2}}{q_{v}}=\frac{\pi^{2}}{\pi \bar{\pi}}=\frac{\pi}{\bar{\pi}}
$$

is a root of unity. This implies that there is a positive (even) integer $m$ such that

$$
\pi^{m}=q_{v}^{m / 2} \in \mathbb{Q}
$$

and therefore $\mathbb{Q}\left(\pi^{m}\right)=\mathbb{Q}$. Let $\kappa(v)_{m}$ be the finite degree $m$ field extension of $\kappa(v)$, which consists of $q_{v}^{m}$ elements. Then $\pi^{m}$ is the Weil $q_{v}^{m}$-number that corresponds to the simple 4-dimensional abelian variety $A(v) \times \kappa(v)_{m}$ over $\kappa(v)_{m}$. Since $\mathbb{Q}\left(\pi^{m}\right)=\mathbb{Q}$, we conclude (as above) that $A(v) \times \kappa(v)_{m}$ has dimension 1 or 2 , which is not the case. 
In both remaining cases the order of the algebra $\operatorname{End}^{0}(A(v)) \otimes_{E} E_{w_{1}}$ in the Brauer group of the $E_{w_{1}} \cong \mathbb{Q}_{\ell}$ is 4 . This implies that $\operatorname{End}^{0}(A(v)) \otimes_{E} E_{w_{1}}$ is neither the matrix algebra of size 4 over $E_{w_{1}}$ nor the matrix algebra of size two over a quaternion algebra over $E_{w_{1}}$. The only remaining possibility is that $\operatorname{End}^{0}(A(v)) \otimes_{E} E_{w_{1}}$ is a division algebra over $E_{w_{1}}$. However, there is again a nonzero (because it sends 1 to 1) $\mathbb{Q}_{\ell}$-algebra homomorphism

$$
D \otimes_{\mathbb{Q}} \mathbb{Q}_{\ell} \rightarrow \operatorname{End}^{0}(A(v)) \otimes_{\mathbb{Q}} \mathbb{Q}_{\ell} \rightarrow \operatorname{End}^{0}(A(v)) \otimes_{E} E_{w_{1}} .
$$

This implies that $\operatorname{End}^{0}(A(v)) \otimes_{E} E_{w_{1}}$ contains zero divisors, which is not the case and we get a contradiction.

Theorem 4.2. If $\ell:=\operatorname{char}(\kappa(v)) \neq p$ then there exists an abelian surface $B(v)$ over $\kappa(v)$ such that $A(v)$ is $\kappa(v)$-isogenous to the square $B(v)^{2}$ of $B(v)$.

Proof. We know that $A(v)$ is not simple and that all $\overline{\kappa(v)}$-endomorphisms of $A(v)$ are defined over $k(v)$. Now let us split $A(v)$ up to a $\kappa(v)$-isogeny into a product of its $\kappa(v)$-isotypic components, using the Poincaré complete reducibility theorem [Lang 1959, Theorem 6 on p. 28 and Theorem 7 on p. 30]. In other words, there is a $\kappa(v)$-isogeny

$$
\mathcal{S}: \prod_{i \in I} A_{i} \rightarrow A(v)
$$

where each $A_{i}$ is a nonzero abelian $\kappa(v)$-subvariety in $A$ such that $\operatorname{End}^{0}\left(A_{i}\right)$ is a simple $\mathbb{Q}$-algebra and $\mathcal{S}$ induces an isomorphism of $\mathbb{Q}$-algebras

$$
\operatorname{End}^{0}(A(v)) \cong \operatorname{End}^{0}\left(\prod_{i \in I} A_{i}\right)=\bigoplus_{i \in I} \operatorname{End}^{0}\left(A_{i}\right) .
$$

This gives us nonzero $\mathbb{Q}$-algebra homomorphisms

$$
D \rightarrow \operatorname{End}^{0}\left(A_{i}\right)
$$

that must be injective, since $D$ is a simple $\mathbb{Q}$-algebra. This implies that each $\operatorname{End}^{0}\left(A_{i}\right)$ is a noncommutative simple $\mathbb{Q}$-algebra, whose $\mathbb{Q}$-dimension is divisible by 8 . In particular, all $\operatorname{dim}\left(A_{i}\right) \geq 2$ and therefore $I$ consists of, at most, 2 elements, since

$$
\sum_{i \in I} \operatorname{dim}\left(A_{i}\right)=\operatorname{dim}(A(v))=4
$$

Since all $\overline{\kappa(v)}$-endomorphisms of $A(v)$ are defined over $k(v)$, all $\overline{\kappa(v)}$-endomorphisms of $A_{i}$ are also defined over $\kappa(v)$; in addition, if $i$ and $j$ are distinct elements of $I$, then every $\overline{\kappa(v)}$-homomorphism between $A_{i}$ and $A_{j}$ is 0 .

If we have $\operatorname{dim}\left(A_{i}\right)=2$ for some $i$ then either $A_{i}$ is isogenous to a square of a supersingular elliptic curve or $A_{i}$ is an absolutely simple abelian surface. However, 
each absolutely simple abelian surface over a finite field is either ordinary (i.e., the slopes of its Newton polygon are 0 and 1, both of length 2) or almost ordinary (i.e., the slopes of its Newton polygon are 0 and 1 , both of length 1 , and $\frac{1}{2}$ with length 2 ): this assertion is well known and follows easily from [Zarhin 2015, Remark 4.1 on p. 2088]. However, in both (ordinary and almost ordinary) cases the endomorphism algebra of a simple abelian variety is commutative [Oort 1992, Lemma 2.3 on p. 136]. This implies that if $\operatorname{dim}\left(A_{i}\right)=2$ then $A_{i}$ is $\kappa(v)$-isogenous to a square of a supersingular elliptic curve. However, if $I$ consists of two elements, say $i$ and $j$, then it follows that both $A_{i}$ and $A_{j}$ are 2-dimensional and therefore both isogenous to a square of a supersingular elliptic curve. This implies that $A_{i}$ and $A_{j}$ are isotypic and therefore $A$ itself is isotypic and we get a contradiction, i.e., none of the $A_{i}$ has dimension 2. It is also clear that if $\operatorname{dim}\left(A_{i}\right)=3$ then $\operatorname{dim}\left(A_{j}\right)=1$, which could not be the case. This implies that $A(v)$ itself is isotypic. It follows that if $\ell=\operatorname{char}(\kappa(v)) \neq p$ then $A(v)$ is $\kappa(v)$-isogenous either to a 4th power of an elliptic curve or to a square of an abelian surface over $\kappa(v)$. (Recall that $A(v)$ is not simple!) In both cases there exists an abelian surface $B(v)$ over $\kappa(v)$, whose square $B(v)^{2}$ is $\kappa(v)$-isogenous to $A(v)$.

Let $B(v)$ be as in Theorem 4.2. One may lift the abelian surface $B(v)$ over $\kappa(v)$ to an abelian surface $B^{v}$ over $K_{v}$, whose reduction is $B(v)$ (see [Oort 1987, Proposition 11.1 on p. 177]). Now if one restricts the action of $G_{K}$ on the $\mathbb{Q}_{r}$-Tate module (here $r$ is any prime different from $\operatorname{char}(\kappa(v))$ )

$$
V_{r}(A)=T_{r}(A) \otimes_{\mathbb{Z}_{r}} \mathbb{Q}_{r}
$$

to the decomposition group $D(v)=G_{K_{v}}$ then the corresponding $G_{K_{v}}$-module $V_{r}(A)$ is unramified (i.e., the inertia group acts trivially) and isomorphic to

$$
V_{r}\left(B^{v}\right) \oplus V_{r}\left(B^{v}\right) .
$$

Theorem 4.3. If $r \neq p$ and $\operatorname{char}(\kappa(v)) \neq r$ then the $G_{K_{v}}$-modules $V_{r}\left(B^{v}\right)$ and $W_{r}(A)$ are isomorphic. In particular, the $G_{K_{v}}$-modules

$$
V_{r}(A)=W_{r}(A) \oplus W_{r}(A)
$$

and

$$
V_{r}\left(B^{v}\right) \oplus V_{r}\left(B^{v}\right)=V_{r}\left(\left(B^{v}\right)^{2}\right)
$$

are isomorphic.

Proof. We know that the $G_{K_{v}}$-modules $W_{r}(A) \oplus W_{r}(A)$ and

$$
V_{r}\left(B^{v}\right) \oplus V_{r}\left(B^{v}\right)
$$

are both isomorphic to $V_{r}(A)$. Since the frobenius endomorphism of $A(v)$ acts on $V_{r}(A)$ as a semisimple linear operator (by a theorem of A. Weil), the $G_{K_{v}}$-module 
$V_{r}(A)$ is semisimple. This implies that the $G_{K_{v}}$-modules $V_{r}\left(B^{v}\right)$ and $W_{r}(A)$ are isomorphic.

For primes $\ell \neq p$, the algebra $D \otimes_{\mathbb{Q}} \mathbb{Q}_{\ell}$ splits, and correspondingly, the representation $V_{\ell}(A)$ splits as $W_{\ell} \oplus W_{\ell}$. Locally, at a place $v \nmid \ell$, we have $W_{\ell} \cong V_{\ell}\left(B^{v}\right)$. However, globally, the representation $W_{\ell}$ does not come from an abelian variety over $K$. Indeed, if the $G_{K}$-module $W_{\ell}$ is isomorphic to $V_{\ell}(B)$ for an abelian variety $B$ over $K$ then $\operatorname{dim}(B)=2$ and the theorem of Faltings implies that there is a nonzero homomorphism of abelian varieties $B \rightarrow A$ over $K$, which is not the case, since the fourfold $A$ is simple. On the other hand, if $v \mid \ell$ then $V_{\ell}(A)$ is a de Rham representation of $G_{K_{v}}$ with weights 0 and -1 , both of multiplicity $\operatorname{dim}(A)=4$. Since a subrepresentation of a de Rham representation is also de Rham, we conclude that $W_{\ell}$ is de Rham. It is also clear that $W_{\ell}$ has the same Hodge-Tate weights as

$$
V_{\ell}(A)=W_{\ell} \oplus W_{\ell}
$$

but the multiplicities should be divided by 2 , i.e., the Hodge-Tate weights of $W_{\ell}$ are 0 and -1 , both of multiplicity 2 .

We thus obtain:

Theorem 4.4. The system of representations $\left\{W_{\ell}\right\}_{\ell \neq p}$ constructed above does not come globally from an abelian variety defined over the field $K$ but for all $v \nmid \ell$ the representation $W_{\ell}$ locally comes from an abelian variety $B^{v} / K_{v}$. In particular, $\left\{W_{\ell}\right\}_{\ell \neq p}$ is a weakly compatible system of 4 -dimensional $\ell$-adic representations of $G_{K}$.

If $v \mid \ell$ then $W_{\ell}$ is locally a de Rham representation with Hodge-Tate weights 0 and -1 , both of multiplicity 2 .

Remark. By a theorem of Faltings [1983], the $G_{K}$-module $V_{\ell}(A)$ is semisimple and therefore its submodule $W_{\ell}$ is also semisimple. On the other hand, we know that the centralizer

$$
\operatorname{End}_{G_{K}}\left(W_{\ell}\right)=k \otimes_{\mathbb{Q}} \mathbb{Q}_{\ell} \neq \mathbb{Q}_{\ell} ;
$$

in particular, none of $W_{\ell}$ is absolutely irreducible. In what follows we construct an example of a weakly compatible system (for all $\ell \neq p$ ) of absolutely irreducible de Rham representations that does not come globally from an abelian variety over a number field. However, we do not know whether it comes locally from abelian varieties.

Let $p$ be a prime and $H$ be a definite quaternion algebra over $\mathbb{Q}$ that is ramified exactly at $p$ and $\infty$. In particular, for each prime $\ell \neq p$ we have a $\mathbb{Q}_{\ell}$-algebra isomorphism

$$
H \otimes_{\mathbb{Q}} \mathbb{Q}_{\ell} \cong \mathbb{M}_{2}\left(\mathbb{Q}_{\ell}\right)
$$


Let $g \geq 4$ be an even integer. According to Shimura [1963] (see also the case of Type III $\left(e_{0}=1\right)$ with $m=g / 2$ in [Oort 1988, Table 8.1 on p. 498] and [Oort and Zarhin 1995, table on p. 23]), there exists a complex $g$-dimensional abelian variety $X$, whose endomorphism algebra $\operatorname{End}^{0}(X)$ is isomorphic to $H$. The same arguments as above (related to $D$ ) prove that there exists a $g$-dimensional abelian variety $B$ over a certain number field $K$ such that all endomorphisms of $B$ are defined over $K$ and $\operatorname{End}^{0}(B) \cong H$. In particular, $B$ is absolutely simple. By the theorem of Faltings, if $\ell$ is a prime then the $G_{K}$-module $V_{\ell}(B)$ is semisimple and

$$
\operatorname{End}_{G_{K}}\left(V_{\ell}(B)\right)=H \otimes_{\mathbb{Q}} \mathbb{Q}_{\ell} .
$$

In particular, if $\ell \neq p$ then $\operatorname{End}_{G_{K}}\left(V_{\ell}(B)\right) \cong \mathbb{M}_{2}\left(\mathbb{Q}_{\ell}\right)$ and therefore there are two isomorphic $\mathbb{Q}_{\ell}\left[G_{K}\right]$-submodules $U_{1, \ell}(B)$ and $U_{2, \ell}(B)$ in $V_{\ell}(B)$ such that

$$
V_{\ell}(B)=U_{1, \ell}(B) \oplus U_{2, \ell}(B) \cong U_{1, \ell}(B) \oplus U_{1, \ell}(B) \cong U_{2, \ell}(B) \oplus U_{2, \ell}(B) .
$$

If we denote by $U_{\ell}$ the $\mathbb{Q}_{\ell}\left[G_{K}\right]$-module $U_{1, \ell}(B)$ then $\operatorname{dim}_{\mathbb{Q}_{\ell}}\left(U_{\ell}\right)=g$ and we get an isomorphism of $\mathbb{Q}_{\ell}\left[G_{K}\right]$-modules

$$
V_{\ell}(B) \cong U_{\ell} \oplus U_{\ell} .
$$

Clearly, the submodule $U_{\ell}$ is semisimple and

$$
\mathbb{M}_{2}\left(\mathbb{Q}_{\ell}\right)=H \otimes_{\mathbb{Q}} \mathbb{Q}_{\ell}=\operatorname{End}_{G_{K}}\left(V_{\ell}(B)\right)=\mathbb{M}_{2}\left(\operatorname{End}_{G_{K}}\left(U_{\ell}\right)\right) .
$$

This implies that $\operatorname{End}_{G_{K}}\left(U_{\ell}\right)=\mathbb{Q}_{\ell}$, i.e., the $\ell$-adic (sub)representation

$$
G_{K} \rightarrow \operatorname{Aut}_{\mathbb{Q}_{\ell}}\left(U_{\ell}\right) \cong \mathrm{GL}_{g}\left(\mathbb{Q}_{\ell}\right)
$$

is absolutely irreducible. Clearly, for each $\sigma \in G_{K}$ its characteristic polynomial with respect to the action on $V_{\ell}(B)$ is the square of its characteristic polynomial with respect to the action on $U_{\ell}$. This implies that if $v$ is an nonarchimedean place $v$ of $K$ where $B$ has good reduction then for all primes $\ell \neq p$ such that $v \nmid \ell$ the characteristic polynomial of the frobenius element at $v$ with respect to its action on $U_{\ell}$ has rational coefficients and does not depend on $\ell$. In other words, $U_{\ell}$ is a weakly compatible system of (absolutely irreducible) $\ell$-adic representations. As above, locally for each $v \mid \ell$ the $G_{K_{v}}$-module $V_{\ell}(B)$ is de Rham with Hodge weights 0 and -1 with weights $g$, which implies that $U_{\ell}$ is also de Rham with the same Hodge-Tate weights, whose multiplicities are $g / 2$.

Theorem 4.5. The weakly compatible system of g-dimensional absolutely irreducible representations $\left\{U_{\ell}\right\}_{\ell \neq p}$ constructed above does not come globally from an abelian variety defined over the field $K$.

If $v \mid \ell$ then $U_{\ell}$ is locally a de Rham representation with Hodge-Tate weights 0 and -1 , both of multiplicity $g / 2$. 
Proof. We claim that none of $U_{\ell}$ comes out from an abelian variety over $K$. Indeed, if there is an abelian variety $C$ over $K$ such that the $G_{K}$-modules $V_{\ell}(C)$ and $U_{\ell}$ are isomorphic then $\operatorname{dim}(C)=g / 2$ and the theorem of Faltings implies the existence of a nonzero homomorphism $C \rightarrow B$, which contradicts the simplicity of $g$-dimensional $B$.

\section{Moduli of curves}

The moduli space of smooth projective curves of genus $g$ is denoted by $\mathcal{M}_{g}$. It is also an orbifold and we will consider its fundamental group as such. For definitions see [Hain 2011]. It is defined over $\mathbb{Q}$ and thus we can consider it over an arbitrary number field $K$. As per our earlier conventions, $\overline{\mathcal{M}}_{g}$ is the base change of $\mathcal{M}_{g}$ to an algebraic closure of $\mathbb{Q}$ and not a compactification.

Let $X$ be a curve of genus $g$ defined over $K$. There is a map (an arithmetic analogue of the Dehn-Nielsen-Baer theorem, see [Matsumoto and Tamagawa 2000], in particular, Lemma 2.1) $\rho: \pi_{1}\left(\mathcal{M}_{g}\right) \rightarrow \operatorname{Out}\left(\pi_{1}(\bar{X})\right)$. This follows by considering the universal curve $\mathcal{C}_{g}$ of genus $g$ together with the map $\mathcal{C}_{g} \rightarrow \mathcal{M}_{g}$, so $X$ can be viewed as a fiber of this map. This gives rise to the fibration exact sequence

$$
1 \rightarrow \pi_{1}(\bar{X}) \rightarrow \pi_{1}\left(\mathcal{C}_{g}\right) \rightarrow \pi_{1}\left(\mathcal{M}_{g}\right) \rightarrow 1
$$

and the action of $\pi_{1}\left(\mathcal{C}_{g}\right)$ on $\pi_{1}(\bar{X})$ gives $\rho$. Now, $X$, viewed as a point on $\mathcal{M}_{g}(K)$, gives a map $\sigma_{\mathcal{M}_{g} / K}(X): G_{K} \rightarrow \pi_{1}\left(\mathcal{M}_{g}\right)$. As pointed out in [Matsumoto and Tamagawa 2000], $\rho \circ \sigma_{\mathcal{M}_{g} / K}(X)$ induces a map $G_{K} \rightarrow \operatorname{Out}\left(\pi_{1}(\bar{X})\right)$ which is none other than the map obtained from the exact sequence (1) by letting $\pi_{1}(X)$ act on $\pi_{1}(\bar{X})$ by conjugation. Combining this with Theorem 2.1 (Mochizuki) gives:

Theorem 5.1. For any field $K$ contained in a finite extension of a $p$-adic field, the section map $\sigma_{\mathcal{M}_{g} / K}$ is injective.

The following result confirms a conjecture of Stoll [2007] if we assume that $\sigma_{\mathcal{M}_{g} / K}$ surjects onto $S_{0}\left(K, \mathcal{M}_{g}\right)$.

Theorem 5.2. Assume that $\sigma_{\mathcal{M}_{g} / K}\left(\mathcal{M}_{g}(K)\right)=S_{0}\left(K, \mathcal{M}_{g}\right)$ for all $g>1$ and all number fields $K$. Then $\sigma_{X / K}(X(K))=S(K, X)$ for all smooth projective curves of genus at least two and all number fields $K$.

Proof. For any algebraic curve $X / K$ there is a nonconstant map $X \rightarrow \mathcal{M}_{g}$ with image $Y$, say, for some $g$, defined over an extension $L$ of $K$, given by the KodairaParshin construction. This gives a map $\gamma: \pi_{1}(X \otimes L) \rightarrow \pi_{1}\left(\mathcal{M}_{g} \otimes L\right)$, over $L$. Let $s \in S(K, X)$, then $\gamma \circ\left(\left.s\right|_{G_{L}}\right) \in S_{0}\left(L, \mathcal{M}_{g}\right)$ and the assumption of the theorem yields that $\gamma \circ\left(\left.s\right|_{G_{L}}\right)=\sigma_{\mathcal{M}_{g} / L}(P), P \in \mathcal{M}_{g}(L)$. We can combine this with the injectivity of $\sigma_{\mathcal{M}_{g} / K_{v}}$ (Mochizuki's theorem) to deduce that in fact $P \in Y\left(L_{v}\right) \cap \mathcal{M}_{g}(L)=Y(L)$. We can consider the pullback to $X$ of the Galois orbit of $P$, which gives us a zero 
dimensional scheme in $X$ having points locally everywhere and, moreover, being unobstructed by every abelian cover coming from an abelian cover of $X$. By the work of Stoll [2007, Proposition 5.2], we conclude that $X$ has a rational point corresponding to $s$.

\section{Acknowledgements}

Voloch would like to thank J. Achter, D. Harari, E. Ozman, T. Schlank, and J. Starr for comments and information. He would also like to thank the Simons Foundation (grant \#234591) and the Centre Bernoulli at EPFL for financial support.

Zarhin is grateful to Frans Oort, Ching-Li Chai and Jiangwei Xue for helpful discussions and to the Simons Foundation for financial and moral support (via grant \#246625 to Yuri Zarkhin). Part of this work was done in May-June 2015 when he was visiting Department of Mathematics of the Weizmann Institute of Science (Rehovot, Israel). The final version of this paper was prepared in May-June 2016 when he was a visitor at the Max-Planck-Institut für Mathematik (Bonn, Germany). The hospitality and support of both institutes is gratefully acknowledged.

We are very grateful to the anonymous referees, whose careful readings and comments have greatly improved the readability of this paper. We would also like to thank W. Sawin for comments.

\section{References}

[André 2004] Y. André, Une introduction aux motifs (motifs purs, motifs mixtes, périodes), Panoramas et Synthèses 17, Société Mathématique de France, Paris, 2004. MR 2115000

[Bass et al. 1964] H. Bass, M. Lazard, and J.-P. Serre, "Sous-groupes d'indice fini dans SL(n, Z)", Bull. Amer. Math. Soc. 70 (1964), 385-392. MR 0161913 Zbl 0232.20086

[Brinon and Conrad 2009] O. Brinon and B. Conrad, "CMI Summer School Notes on $p$-adic Hodge Theory”, preprint, Clay Mathematics Institute, 2009, Available at http://www.claymath.org/ galois-representations. Galois Representations (Honolulu, 2009).

[Deligne 1971] P. Deligne, "Formes modulaires et représentations $l$-adiques", exposé no. 355, 139-172 in Séminaire Bourbaki 1968/69, Lecture Notes in Math. 175, Springer, Berlin, 1971. MR 3077124

[Faltings 1983] G. Faltings, "Endlichkeitssätze für abelsche Varietäten über Zahlkörpern”, Invent. Math. 73:3 (1983), 349-366. MR 718935 Zbl 0588.14026

[Faltings 1984] G. Faltings, "Complements to Mordell”, pp. 203-227 in Rational points (Bonn, 1983/1984), edited by G. Faltings and G. Wüstholz, Aspects Math. E6, Friedr. Vieweg \& Sohn, Braunschweig, 1984. MR 766574

[Faltings 1989] G. Faltings, "Crystalline cohomology and p-adic Galois-representations", pp. 25-80 in Algebraic analysis, geometry, and number theory (Baltimore, MD, 1988), edited by J.-I. Igusa, Johns Hopkins Univ. Press, Baltimore, MD, 1989. MR 1463696 Zbl 0805.14008

[Fontaine and Mazur 1995] J.-M. Fontaine and B. Mazur, "Geometric Galois representations", pp. 41-78 in Elliptic curves, modular forms, \& Fermat's last theorem, 1st ed. (Hong Kong, 1993), edited by J. Coates, Int. Press, Cambridge, MA, 1995. MR 1363495 Zbl 0839.14011 
[Fontaine and Ouyang 2007] J.-M. Fontaine and Y. Ouyang, "Theory of $p$-adic representations", preprint, 2007, Available at http://www.math.u-psud.fr/ fontaine/recherche.html.

[Grothendieck 1997a] A. Grothendieck, "Brief an G. Faltings", pp. 49-58 in Geometric Galois actions, 1, edited by L. Schneps and P. Lochak, London Math. Soc. Lecture Note Ser. 242, Cambridge Univ. Press, 1997. MR 1483108 Zbl 0901.14002

[Grothendieck 1997b] A. Grothendieck, "Esquisse d'un programme”, pp. 5-48 in Geometric Galois actions, 1, edited by L. Schneps and P. Lochak, London Math. Soc. Lecture Note Ser. 242, Cambridge Univ. Press, 1997. MR 1483107 Zbl 0901.14001

[Hain 2011] R. Hain, "Rational points of universal curves", J. Amer. Math. Soc. 24:3 (2011), 709-769. MR 2784328 Zbl 1225.14016

[Harari and Stix 2012] D. Harari and J. Stix, "Descent obstruction and fundamental exact sequence", pp. 147-166 in The arithmetic of fundamental groups: PIA 2010, edited by J. Stix, Contrib. Math. Comput. Sci. 2, Springer, Heidelberg, 2012. MR 3220518 Zbl 1315.14032

[Harari and Voloch 2010] D. Harari and J. F. Voloch, "The Brauer-Manin obstruction for integral points on curves", Math. Proc. Cambridge Philos. Soc. 149:3 (2010), 413-421. MR 2726726 Zbl 1280.11038

[Helm and Voloch 2011] D. Helm and J. F. Voloch, "Finite descent obstruction on curves and modularity”, Bull. Lond. Math. Soc. 43:4 (2011), 805-810. MR 2820165 Zbl 1235.11062

[Ihara and Nakamura 1997] Y. Ihara and H. Nakamura, "Some illustrative examples for anabelian geometry in high dimensions", pp. 127-138 in Geometric Galois actions, 1, edited by L. Schneps and P. Lochak, London Math. Soc. Lecture Note Ser. 242, Cambridge Univ. Press, 1997. MR 1483114 Zbl 0919.14011

[Jannsen 1992] U. Jannsen, "Motives, numerical equivalence, and semi-simplicity", Invent. Math. 107:3 (1992), 447-452. MR 1150598 Zbl 0762.14003

[Lang 1959] S. Lang, Abelian varieties, Interscience Tracts in Pure and Appl. Math. 7, Interscience, New York, 1959. Reprinted Springer 1983. MR 0106225 Zbl 0099.16103

[Matsumoto and Tamagawa 2000] M. Matsumoto and A. Tamagawa, "Mapping-class-group action versus Galois action on profinite fundamental groups", Amer. J. Math. 122:5 (2000), 1017-1026. MR 1781929 Zbl 0993.12002

[Mazur 1999] B. Mazur, "Open problems in number theory", pp. 199-203 in Current developments in mathematics (Cambridge, MA, 1997), edited by R. Bott et al., Int. Press, Boston, 1999. MR 1700300

[Mennicke 1965] J. L. Mennicke, "Finite factor groups of the unimodular group", Ann. of Math. (2) 81 (1965), 31-37. MR 0171856 Zbl 0135.06504

[Mochizuki 1996] S. Mochizuki, "The profinite Grothendieck conjecture for closed hyperbolic curves over number fields", J. Math. Sci. Univ. Tokyo 3:3 (1996), 571-627. MR 1432110 Zbl 0889.11020

[Mochizuki 2003] S. Mochizuki, "Topics surrounding the anabelian geometry of hyperbolic curves", pp. 119-165 in Galois groups and fundamental groups, edited by L. Schneps, Math. Sci. Res. Inst. Publ. 41, Cambridge Univ. Press, 2003. MR 2012215 Zbl 1053.14029

[Mumford 1966] D. Mumford, "On the equations defining abelian varieties, I", Invent. Math. 1 (1966), 287-354. MR 0204427 Zbl 0219.14024

[Mumford 1970] D. Mumford, Abelian varieties, Tata Inst. Fund. Res. Stud. Math. 5, Oxford University Press, London, 1970. MR 0282985 Zbl 0223.14022

[Noot 1995] R. Noot, "Abelian varieties - Galois representation and properties of ordinary reduction", Compositio Math. 97:1-2 (1995), 161-171. MR 1355123 Zbl 0868.14021 
[Oort 1987] F. Oort, "Lifting algebraic curves, abelian varieties, and their endomorphisms to characteristic zero", pp. 165-195 in Algebraic geometry: Bowdoin, 1985 (Brunswick, ME, 1985), edited by S. J. Bloch, Proc. Sympos. Pure Math. 46, Amer. Math. Soc., Providence, RI, 1987. MR 927980 Zbl 0645.14017

[Oort 1988] F. Oort, "Endomorphism algebras of abelian varieties", pp. 469-502 in Algebraic geometry and commutative algebra, vol. II, edited by H. Hijikata et al., Kinokuniya, Tokyo, 1988. MR 977774 Zbl 0697.14029

[Oort 1992] F. Oort, "CM-liftings of abelian varieties”, J. Algebraic Geom. 1:1 (1992), 131-146. MR 1129842 Zbl 0803.14025

[Oort and Zarhin 1995] F. Oort and Y. Zarhin, "Endomorphism algebras of complex tori", Math. Ann. 303:1 (1995), 11-29. MR 1348352 Zbl 0858.14024

[Pál 2011] A. Pál, “The real section conjecture and Smith's fixed-point theorem for pro-spaces”, $J$. Lond. Math. Soc. (2) 83:2 (2011), 353-367. MR 2776641 Zbl 1263.14056

[Patrikis 2012] S. Patrikis, Variations on a theorem of Tate, Ph.D. thesis, Princeton University, 2012, Available at http://search.proquest.com/docview/1040698945. MR 3078437 arXiv 1207.6724

[Ribet 1976] K. A. Ribet, "Galois action on division points of Abelian varieties with real multiplications", Amer. J. Math. 98:3 (1976), 751-804. MR 0457455 Zbl 0348.14022

[Serre 1989] J.-P. Serre, Abelian l-adic representations and elliptic curves, 2nd ed., Addison-Wesley, Redwood City, CA, 1989. MR 1043865 Zbl 0709.14002

[SGA $4 \frac{1}{2}$ 1977] P. Deligne, Cohomologie étale (Séminaire de Géométrie Algébrique du Bois Marie), Lecture Notes in Math. 569, Springer, Berlin, 1977. MR 0463174 Zbl 0345.00010

[SGA 7 I 1972] A. Grothendieck, "Exposé IX: modèles de Néron et monodromie", pp. 313-523 in Groupes de monodromie en géométrie algébrique, I (Séminaire de Géométrie Algébrique du Bois Marie 1967-1969), edited by A. Grothendieck et al., Lecture Notes in Math. 288, Springer, Berlin, 1972. MR 0354656 Zbl 0237.00013

[Shimura 1963] G. Shimura, "On analytic families of polarized abelian varieties and automorphic functions”, Ann. of Math. (2) 78 (1963), 149-192. MR 0156001 Zbl 0142.05402

[Silverberg 1992] A. Silverberg, "Fields of definition for homomorphisms of abelian varieties", $J$. Pure Appl. Algebra 77:3 (1992), 253-262. MR 1154704 Zbl 0808.14037

[Silverberg and Zarhin 1995] A. Silverberg and Y. G. Zarhin, "Semistable reduction and torsion subgroups of abelian varieties", Ann. Inst. Fourier (Grenoble) 45:2 (1995), 403-420. MR 1343556 Zbl 0818.14017

[Stix 2013] J. Stix, Rational points and arithmetic of fundamental groups: evidence for the section conjecture, Lecture Notes in Math. 2054, Springer, Heidelberg, 2013. MR 2977471 Zbl 1272.14003

[Stoll 2007] M. Stoll, "Finite descent obstructions and rational points on curves", Algebra Number Theory 1:4 (2007), 349-391. MR 2368954 Zbl 1167.11024

[Tate 1966] J. Tate, "Endomorphisms of abelian varieties over finite fields", Invent. Math. 2 (1966), 134-144. MR 0206004 Zbl 0147.20303

[Tate 1971] J. Tate, "Classes d'isogénie des variétés abéliennes sur un corps fini (d'après T. Honda)", exposé no. 352, 95-110 in Séminaire Bourbaki 1968/69, Lecture Notes in Math. 175, Springer, Berlin, 1971. MR 3077121 Zbl 0212.25702

[Tate 1994] J. Tate, "Conjectures on algebraic cycles in $l$-adic cohomology", pp. 71-83 in Motives (Seattle, WA, 1991), edited by U. Jannsen et al., Proc. Sympos. Pure Math. 55, Amer. Math. Soc., Providence, RI, 1994. MR 1265523 Zbl 0814.14009 
[Voloch 2012] J. F. Voloch, "Finite descent obstruction for curves over function fields", Bull. Braz. Math. Soc. (N.S.) 43:1 (2012), 1-6. MR 2909919 Zbl 1300.11073

[Zarhin 1985] Y. G. Zarhin, "A finiteness theorem for unpolarized abelian varieties over number fields with prescribed places of bad reduction", Invent. Math. 79:2 (1985), 309-321. MR 778130 Zbl 0557.14024

[Zarhin 2015] Y. G. Zarhin, "Eigenvalues of Frobenius endomorphisms of abelian varieties of low dimension”, J. Pure Appl. Algebra 219:6 (2015), 2076-2098. MR 3299720 Zbl 06398749

[Zoonekynd 2001] V. Zoonekynd, “The fundamental group of an algebraic stack”, preprint, 2001. arXiv math/0111071

Communicated by Brian Conrad

Received 2015-07-14 Revised 2016-05-26 Accepted 2016-06-25

patrikis@math.utah.edu Department of Mathematics, University of Utah, 155 S 1400 E, Salt Lake City, UT 84112, United States

felipe.voloch@canterbury.ac.nz School of Mathematics and Statistics, University of Canterbury, Private Bag 4800, Christchurch 8140, New Zealand

voloch@math.utexas.edu Department of Mathematics, University of Texas, Austin, TX 78712, United States

zarhin@math.psu.edu

Department of Mathematics, Pennsylvania State University, University Park, PA 16802, United States 


\section{Algebra \& Number Theory}

msp.org/ant

\section{EDITORS}

MANAGING EDITOR

Bjorn Poonen

Massachusetts Institute of Technology

Cambridge, USA

\author{
EDITORIAL BOARD CHAIR \\ David Eisenbud \\ University of California \\ Berkeley, USA
}

BOARD OF EDITORS

$\begin{aligned} \text { Dave Benson } & \text { University of Aberdeen, Scotland } & \text { Susan Montgomery } & \text { University of Southern California, USA } \\ \text { Richard E. Borcherds } & \text { University of California, Berkeley, USA } & \text { Shigefumi Mori } & \text { RIMS, Kyoto University, Japan } \\ \text { John H. Coates } & \text { University of Cambridge, UK } & \text { Raman Parimala } & \text { Emory University, USA } \\ \text { J-L. Colliot-Thélène } & \text { CNRS, Université Paris-Sud, France } & \text { Jonathan Pila } & \text { University of Oxford, UK } \\ \text { Brian D. Conrad } & \text { Stanford University, USA } & \text { Anand Pillay } & \text { University of Notre Dame, USA } \\ \text { Hélène Esnault } & \text { Freie Universität Berlin, Germany } & \text { Victor Reiner } & \text { University of Minnesota, USA } \\ \text { Hubert Flenner } & \text { Ruhr-Universität, Germany } & \text { Peter Sarnak } & \text { Princeton University, USA } \\ \text { Sergey Fomin } & \text { University of Michigan, USA } & \text { Joseph H. Silverman } & \text { Brown University, USA } \\ \text { Edward Frenkel } & \text { University of California, Berkeley, USA } & \text { Michael Singer } & \text { North Carolina State University, USA } \\ \text { Andrew Granville } & \text { Université de Montréal, Canada } & \text { Vasudevan Srinivas } & \text { Tata Inst. of Fund. Research, India } \\ \text { Joseph Gubeladze } & \text { San Francisco State University, USA } & \text { J. Toby Stafford } & \text { University of Michigan, USA } \\ \text { Roger Heath-Brown } & \text { Oxford University, UK } & \text { Ravi Vakil } & \text { Stanford University, USA } \\ \text { Craig Huneke } & \text { University of Virginia, USA } & \text { Michel van den Bergh } & \text { Hasselt University, Belgium } \\ \text { Kiran S. Kedlaya } & \text { Univ. of California, San Diego, USA } & \text { Marie-France Vignéras } & \text { Université Paris VII, France } \\ \text { János Kollár } & \text { Princeton University, USA } & \text { Kei-Ichi Watanabe } & \text { Nihon University, Japan } \\ \text { Yuri Manin } & \text { Northwestern University, USA } & \text { Efim Zelmanov } & \text { University of California, San Diego, USA } \\ \text { Philippe Michel } & \text { École Polytechnique Fédérale de Lausanne } & \text { Shou-Wu Zhang } & \text { Princeton University, USA }\end{aligned}$

PRODUCTION

production@msp.org

Silvio Levy, Scientific Editor

See inside back cover or msp.org/ant for submission instructions.

The subscription price for 2016 is US $\$ 290$ /year for the electronic version, and $\$ 485 /$ year (+\$55, if shipping outside the US) for print and electronic. Subscriptions, requests for back issues and changes of subscribers address should be sent to MSP.

Algebra \& Number Theory (ISSN 1944-7833 electronic, 1937-0652 printed) at Mathematical Sciences Publishers, 798 Evans Hall \#3840, c/o University of California, Berkeley, CA 94720-3840 is published continuously online. Periodical rate postage paid at Berkeley, CA 94704, and additional mailing offices.

ANT peer review and production are managed by EditFLow ${ }^{\circledR}$ from MSP.

\section{PUBLISHED BY}

- mathematical sciences publishers

nonprofit scientific publishing

http://msp.org/

() 2016 Mathematical Sciences Publishers 


\section{Algebra \& Number Theory}

Volume $10 \quad$ No. $6 \quad 2016$

Modular elliptic curves over real abelian fields and the generalized Fermat equation $x^{2 \ell}+y^{2 m}=z^{p}$

SAMUELE ANNI and SAMIR SIKSEK

Geometry and stability of tautological bundles on Hilbert schemes of points

DAVID STAPLETON

Anabelian geometry and descent obstructions on moduli spaces

Stefan PATRIKIS, José Felipe Voloch and Yuri G. Zarhin

On the local Tamagawa number conjecture for Tate motives over tamely ramified fields JAY DAIGLE and MATTHIAS FLACH

Heegner divisors in generalized Jacobians and traces of singular moduli

JAN HENDRIK BRUINIER and YINGKUN LI

On 2-dimensional 2-adic Galois representations of local and global fields VYTAUTAS PAŠKŪNAS

A probabilistic Tits alternative and probabilistic identities

Michael LARSEN and ANER SHALEV 